\title{
Euclidean vacuum mode functions for a scalar field on open de Sitter space
}

\author{
Misao Sasaki, Takahiro Tanaka and Kazuhiro Yamamoto \\ Department of Physics, Kyoto University \\ Kyoto 606-01, Japan
}

\begin{abstract}
Motivated by recent studies of the one-bubble inflationary universe scenario that predicts a low density, negative curvature universe, we investigate the Euclidean vacuum mode functions of a scalar field in a spatially open chart of de Sitter space which is foliated by hyperbolic time slices. When we consider the possibility of an open inflationary universe, we are faced with the problem of the initial condition for the quantum fluctuations of the inflaton field, because the inflationary era should not last too long to lose every information of the initial condition. In the one-bubble scenario in which an open universe is created in an exponentially expanding false vacuum universe triggered by quantum decay of false vacuum, it seems natural that the initial state is the de Sitter-invariant Euclidean vacuum. Here we present explicit expressions for the Euclidean vacuum mode functions in the open chart for a scalar field with arbitrary mass and curvature coupling.

03.65.Sq, 03.70.+k and 98.80.Cq.
\end{abstract}

\section{INTRODUCTION}

Recently there appeared not a few observations which suggest our universe has negative curvature, i.e., $\Omega_{0} \sim 0.1$ [1]. Accordingly, papers comparing theoretical predictions of open universe models with observational data such as COBE [2] have been appearing in the context of inflationary universe models [3], in cosmological models with topological defects [4] or in a general context by assuming a power-law type primordial density perturbation spectrum [5]. However, in the standard inflationary universe paradigm, it is generally believed that an open FRW universe with small perturbations is hard to be realized in a consistent manner [6].

One possible consistent scenario is the creation of an open universe from an exponentially expanding false vacuum dominated universe [7 10]. Originally this idea was proposed by Gott 11]. In the standard inflationary universe scenario, the horizon problem is solved by a large amount of expansion of space. A homogeneous patch initially of a horizon size expands exponentially and our present horizon size will be inside such a homogeneous patch. However in this context, the flatness (or entropy) problem is solved at the same time when the horizon problem is solved. Therefore the spatial curvature at present time is inevitably decreased by the expansion of universe. This is the problem of the standard scenario of inflation if we attempt to construct an open universe model with $1-\Omega_{0}=O(1)$. On the other hand, if we consider a bubble nucleation in the sea of false vacuum which is described by de Sitter space, the interior of a bubble has the $O(3,1)$ invariance owing to the $O(4)$ symmetry of the Euclidean bounce solution which represents the tunneling process [12]. Thus the horizon problem is automatically solved. Though the spacetime has the exact $O(3,1)$ invariance in the lowest order description, quantum fluctuations around the classical background may give rise to cosmological density perturbations to explain the large scale structure of the universe. However if the vacuum energy never becomes a dominant component of the cosmic energy density after nucleation, the universe will be curvature dominated from the beginning and never recover to a hot FRW universe. Thus the entropy problem cannot be solved. To solve this problem, a secondary inflation in the bubble is required. The essential difference of this scenario from the standard scenario is that a horizon size patch whose size is approximately equal to the curvature scale at the onset of the secondary inflation may not become much larger than the present horizon scale. In such a case, we will have a sufficiently homogeneous open universe at present. This implies that memories of the quantum state of the universe at the beginning of the secondary inflation will not be erased but will directly affect the observed large scale temperature and density fluctuations [8] 10 .

\footnotetext{
*Address after January 1, 1995: Department of Earth and Space Science, Osaka University, Toyonaka 560.
} 
Therefore the quantum state of a field, $\phi$, inside the nucleated bubble, especially that of the inflaton field of the secondary inflation, is to be examined. A pioneering study of this subject was done by Rubakov [13] and a formalism which respects the $O(4)$ symmetry of the tunneling background was developed by Vachaspati and Vilenkin [14]. Meanwhile we developed a formalism based on the multi-dimensional tunneling wave function [15] and applied it to the $O(4)$-symmetric bubble nucleation without gravitational effects [16] and with gravitational effects [17]. However, all of these previous works remained in a rather formal level in the sense that techniques to investigate the quantum state which have practical applicability to a general situation have not been developed. Recently, we have succeeded in giving a general and practical method to obtain the quantum state inside a nucleated bubble in flat space [18] and its application to the case of the quantum state of the tunneling field itself is in progress [19]. Thus an extension of this method to the case when gravity comes into play is now to be formulated.

As a first step, we consider the case when the gravitational back reaction effect can be neglected so that the background metric may be fixed to that of de Sitter space. Then the spacetime inside a bubble is described by a spatially open chart of the de Sitter space and the inflaton field will take a constant value on a hypersurface of the spatially open time slicing. Further, if the tunneling field which causes the bubble nucleation has no interaction with $\phi$, the quantum state of $\phi$ will not be affected by the tunneling process at all. Then, provided that the quantum state of $\phi$ before tunneling is in the Euclidean vacuum, which should be a good approximation if the preceding false vacuum inflation lasted long enough, the quantum state inside a nucleated bubble will remain so. Hence, it is required to describe the quantum state by the mode functions in an open chart of de Sitter space. Once we know a method to obtain the Euclidean vacuum mode functions in the open chart, it should be fairly straightforward to extend it to the case in which the mass of $\phi$ changes in time due to its coupling to the tunneling field or when the geometry deviates from the exact de Sitter space at later stages.

In a spatially flat or closed chart, the field operator can be relatively easily decomposed into the spatial harmonics and the mode functions corresponding to the Euclidean vacuum are well known [20]. However, the Euclidean vacuum mode functions in a spatially open chart have not been known except for the massless conformally coupled case [21]. Here we give an explicit expression of vacuum mode functions for a scalar field with arbitrary mass and curvature coupling, including the massless minimal coupling limit.

The paper is organized as follows. In Section II, we quantize a scalar field on the hyperbolic time slices which foliate two distinct open charts of de Sitter space (see Fig. 1). We define a vacuum state there by requiring that the positive frequency functions be regular on a hemisphere of the Euclidean de Sitter space where the complexified time coordinate is negative pure imaginary. Then we derive an expression for the Wightman function for this vacuum state in the series form. In Section III, by analyzing the behavior of the Wightman function for thus obtained vacuum state, we show that it is in fact the de Sitter-invariant Euclidean vacuum, the so-called Bunch-Davies vacuum [20], provided that the effective mass of the scalar field is greater than a critical value which corresponds to the conformally coupled massless case. However, we also find that our expression for the Wightman function does not coincide with the Euclidean vacuum one for mass smaller than the critical value. In Section IV, by carefully analyzing this discrepancy in the small mass case, we find that there exist a set of modes which have finite Klein-Gordon norms on regular Cauchy surfaces, say on spatially closed time slices, but which cannot be quantized on the open charts because of the divergent Klein-Gordon norms on the hyperbolic time slices. Then normalizing these modes on a spatially closed hypersurface and analytically continuing them back to the open charts, we obtain a complete description of a scalar field and the Euclidean vacuum there in terms of the orthonormalized positive frequency functions, irrespective of its mass. In Section V, we consider the special cases of a massless conformal scalar and a massless minimal scalar in which the series expression for the Wightman function can be summed up with elementary algebra. The results are found to be in perfect agreement with our analysis for general mass in the preceding sections. In Section VI, we summarize our results and discuss their implications. Finally, Appendix A explicitly evaluates the Klein-Gordon norms of the mode functions on a closed slice for which their norms on a hyperbolic slice are finite, to show their equivalence and Appendix B gives a proof of a mathematical formula which plays a central role in the analysis of the set of modes whose Klein-Gordon norms diverge on a hyperbolic slice.

\section{SCALAR FIELD ON OPEN DE SITTER SPACE}

To begin with, we introduce a coordinate system which covers the whole Euclidean de Sitter space. The fourdimensional Euclidean de Sitter space is a four-sphere and can be embedded in the five-dimensional Euclidean space, $\left(\tilde{x}^{0}, x^{i}\right)$, as a hypersurface which satisfies $H^{-2}=\left(\tilde{x}^{0}\right)^{2}+\sum\left(x^{i}\right)^{2}$, where $H^{-1}$ is the Hubble radius of the de Sitter space. A fundamental coordinate system we use in the Euclidean region is defined as 


$$
\tilde{x}^{0}=\cos \tau \cos \rho, \quad x^{1}=\sin \tau, \quad\left(\begin{array}{l}
x^{2} \\
x^{3} \\
x^{4}
\end{array}\right)=\cos \tau \sin \rho\left(\begin{array}{c}
\cos \theta \\
\sin \theta \cos \varphi \\
\sin \theta \sin \varphi
\end{array}\right) . \quad\left(\begin{array}{c}
-\pi / 2 \leq \tau \leq \pi / 2 \\
0 \leq \rho \leq \pi
\end{array}\right)
$$

Then the metric is represented as

$$
d s_{E}^{2}=H^{-2}\left(d \tau^{2}+\cos ^{2} \tau\left(d \rho^{2}+\sin ^{2} \rho d \Omega^{2}\right)\right) .
$$

Coordinate systems in the Lorentzian region are obtained by the analytic continuation, $\tilde{x}^{0} \rightarrow i x^{0}$. Performing this, we find that the Lorentzian region may be divided into three parts, which we call $R, C$, and $L$, whose coordinates are related to the fundamental ones by the relations,

$$
\begin{aligned}
& \left\{\begin{aligned}
t_{R} & =i(\tau-\pi / 2), & & \left(t_{R} \geq 0\right) \\
r_{R} & =i \rho, & & \left(r_{R} \geq 0\right)
\end{aligned}\right. \\
& \left\{\begin{array}{rlrl}
t_{C} & =\tau, & & \left(\pi / 2 \geq t_{C} \geq-\pi / 2\right) \\
r_{C}=i(\rho-\pi / 2), & & \left(\infty>r_{C}>-\infty\right)
\end{array}\right.
\end{aligned}
$$

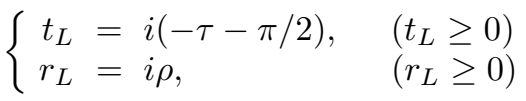

and their metrics are given respectively by

$$
\begin{aligned}
d s_{R}^{2} & =H^{-2}\left(-d t_{R}^{2}+\sinh ^{2} t_{R}\left(d r_{R}^{2}+\sinh ^{2} r_{R} d \Omega^{2}\right)\right), \\
d s_{C}^{2} & =H^{-2}\left(d t_{C}^{2}+\cos ^{2} t_{C}\left(-d r_{C}^{2}+\cosh ^{2} r_{C} d \Omega^{2}\right)\right), \\
d s_{L}^{2} & =H^{-2}\left(-d t_{L}^{2}+\sinh ^{2} t_{L}\left(d r_{L}^{2}+\sinh ^{2} r_{L} d \Omega^{2}\right)\right) .
\end{aligned}
$$

In the above, the regions $R$ and $L$ are connected through $\tau \in(-\pi / 2, \pi / 2)$ along a path over the hemisphere $\Im x^{0}<0$ $\left(\tilde{x}^{0}>0\right)$, say along $\rho=0$, and the region $C$ is joined to this hemisphere at $\rho=\pi / 2$. Note that one may connect the regions $R$ and $L$ in a different way by passing through the other hemisphere $\Im x^{0}>0\left(\tilde{x}^{0}<0\right)$, say along $\rho=\pi$, but we take the former choice given by Eq. (2.3) for later convenience. A schematic picture of how these coordinates cover the spacetime is shown in a conformal diagram in Fig. 1. The regions $R$ and $L$ covered by the coordinates $\left(t_{R}, r_{R}\right)$ and $\left(t_{L}, r_{L}\right)$, respectively, are the two distinct spatially open charts of de Sitter space. We see that the whole Euclidean and Lorentzian de Sitter space is covered by complexifying the variables $\tau$ and $\rho$.

Let us consider a free scalar field in de Sitter space with curvature coupling constant $\xi$. We expand the field operator as

$$
\hat{\phi}(x)=\sum_{\Lambda}\left(\hat{a}_{\Lambda} u_{\Lambda}(x)+\hat{a}_{\Lambda}^{\dagger} \overline{u_{\Lambda}(x)}\right)
$$

where a bar denotes the complex conjugate and $\left\{u_{\Lambda}(x)\right\}$ forms a complete set of mode functions labeled by certain indices $\Lambda$ which satisfy the field equation,

$$
\left[g^{\mu \nu} \nabla_{\mu} \nabla_{\nu}-M_{\text {eff }}^{2}\right] u_{\Lambda}(x)=0 ; \quad M_{\text {eff }}^{2}:=M^{2}+12 \xi H^{2},
$$

and normalized with respect to the Klein-Gordon inner product. The condition, $\hat{a}_{\Lambda}|0\rangle=0$ for any $\Lambda$, determines the vacuum state associated with a specified set of mode functions.

To find the Euclidean vacuum mode functions on the open chart, we write down the field equation (2.7) in terms of the coordinates in either of the regions $R$ or $L$. Since these two regions are completely symmetric we have

$$
\left[\frac{1}{a^{3}(t)} \frac{\partial}{\partial t} a^{3}(t) \frac{\partial}{\partial t}-\frac{H^{-2}}{a^{2}(t)} \mathbf{L}^{2}+\frac{9}{4}-\nu^{2}\right] u_{\Lambda}(t, r, \Omega)=0
$$

where $(t, r)=\left(t_{R}, r_{R}\right)$ or $\left(t_{L}, r_{L}\right)$,

$$
\begin{aligned}
a(t) & =H^{-1} \sinh t, \quad \nu=\sqrt{\frac{9}{4}-\frac{M_{\text {eff }}^{2}}{H^{2}}} \\
\mathbf{L}^{2} & =\frac{1}{\sinh ^{2} r} \frac{\partial}{\partial r}\left(\sinh ^{2} r \frac{\partial}{\partial r}\right)+\frac{1}{\sinh ^{2} r} \mathbf{L}_{\Omega}^{2},
\end{aligned}
$$


and $\mathbf{L}_{\Omega}^{2}$ is the usual Laplacian operator on the unit two-sphere. We write the eigenvalue equation for the operator $-\mathbf{L}^{2}$ on a unit three-dimensional hyperboloid in the form [22],

$$
-\mathbf{L}^{2} Y_{p l m}(r, \Omega)=\left(1+p^{2}\right) Y_{p l m}(r, \Omega) .
$$

The eigenfunction $Y_{p l m}$ which is regular at $r=0$ is given by

$$
\begin{aligned}
Y_{p l m}(r, \Omega) & =f_{p l}(r) Y_{l m}(\Omega) \\
f_{p l}(r) & :=\frac{\Gamma(i p+l+1)}{\Gamma(i p+1)} \frac{p}{\sqrt{\sinh r}} P_{i p-1 / 2}^{-l-1 / 2}(\cosh r) \\
& =(-1)^{l} \sqrt{\frac{2}{\pi}} \frac{\Gamma(-i p+1)}{\Gamma(-i p+l+1)} \sinh ^{l} r \frac{d^{l}}{d(\cosh r)^{l}}\left(\frac{\sin p r}{\sinh r}\right) .
\end{aligned}
$$

where $Y_{l m}(\Omega)$ is the normalized spherical harmonic function on the unit two-sphere, $\Gamma(z)$ is the Gamma function and $P_{\mu}^{\nu}(z)$ is the associated Legendre function of the first kind [23]. The eigenfunctions $Y_{p l m}$ with real positive values of $p$ form an orthonormal complete set for square-integrable functions on the unit three-hyperboloid. They are normalized as

$$
\int_{0}^{\infty} d r \sinh ^{2} r \int d \Omega Y_{p l m}(r, \Omega) \overline{Y_{p^{\prime} l^{\prime} m^{\prime}}(r, \Omega)}=\delta\left(p-p^{\prime}\right) \delta_{l l^{\prime}} \delta_{m m^{\prime}}
$$

We now take the harmonic expansion of the mode functions,

$$
u_{p l m}(t, r, \Omega)=\frac{1}{a(t)} \chi_{p l m}(t) Y_{p l m}(r, \Omega),
$$

and look for a complete set of positive frequency functions $\left\{\chi_{p l m}(t)\right\}$ for the Euclidean vacuum.

As well-known, the positive frequency functions of the Euclidean vacuum are characterized by their regularity on the $\Im x^{0}<0$ hemisphere of the Euclidean de Sitter space. To find such functions, let us first consider the mode functions in the region $R$. The general solution is expressed as a linear combination of the associated Legendre functions $P_{\nu-1 / 2}^{i p}\left(z_{R}\right)$ and $P_{\nu-1 / 2}^{-i p}\left(z_{R}\right)$, where $z_{R}=\cosh t_{R}$. To find out their coefficients, we first ignore the normalization and consider a solution,

$$
\chi_{p}^{(R)}=P_{\nu^{\prime}}^{i p}\left(z_{R}\right)
$$

where we have introduced $\nu^{\prime}:=\nu-1 / 2$ for notational simplicity. Note that this function would be a natural candidate for the positive frequency function if the region $R$ were the whole universe. Imposing the above regularity condition on $\chi_{p}^{(R)}$, we find that the analytic continuation of it to the region $L$ amounts to going through the branch cut $[-1,1]$ of the associated Legendre function $P_{\nu^{\prime}}^{i p}(z)$ from $\Im z<0$ to $\Im z>0$ and then to $z<-1$ on the real axis. Thus in the region $L$ we have

$$
\begin{aligned}
\chi_{p}^{(R)} & =e^{-\pi p} P_{\nu^{\prime}}^{i p}\left(-z_{L}\right) \\
& =e^{\pi\left(-p+i \nu^{\prime}\right)} P_{\nu^{\prime}}^{i p}\left(z_{L}\right)-\frac{2 \sin \pi\left(i p+\nu^{\prime}\right)}{\pi} Q_{\nu^{\prime}}^{i p}\left(z_{L}\right),
\end{aligned}
$$

where $z_{L}=\cosh t_{L}$ and $Q_{\nu}{ }^{\mu}(z)$ is the associated Legendre function of the second kind [23]. Replacing the role of $R$ and $L$, we obtain $\chi_{p}^{(L)}$ in the same way. With this choice of the mode functions, their regularity on the $\Im x^{0}<0$ hemisphere is automatically guaranteed because the harmonic function $Y_{p l m}$ is regular at $r_{R}=r_{L}=\rho=0$, hence regular for $0 \leq \rho \leq \pi / 2$.

As the mode functions we have obtained are not yet normalized, the Klein-Gordon inner products of these mode functions should be calculated to normalize them. The evaluation of the Klein-Gordon inner products must be performed on a Cauchy surface. As the choice of a surface is arbitrary as long as it is a Cauchy surface, we choose it in the following way. It consists of the three parts, (I),(II) and (III), where (I) is the $r_{R}<r_{\text {max }}$ part of $t_{R}=$ constant hypersurface for a large $r_{\max }$, (II) is the one in which $R$ is replaced by $L$, and (III) is a bridge connecting these two isolated parts. A schematic picture of this Cauchy surface is drawn in Fig. 2. If the contribution from the integral over the surface (III) can be neglected for sufficiently large $r_{\max }$, the Klein-Gordon inner product reduces to the summation of integrals over the surfaces (I) and (II). In such a case we have 


$$
\begin{aligned}
\left\langle\frac{\chi_{1}(t)}{a(t)} Y_{p l m}(r, \Omega)\right. & \left., \frac{\chi_{2}(t)}{a(t)} Y_{p^{\prime} l^{\prime} m^{\prime}}(r, \Omega)\right\rangle \\
& =\left[\left\{i\left(z_{R}^{2}-1\right)\left(\frac{d \chi_{1}}{d z_{R}} \overline{\chi_{2}}-\chi_{1} \frac{\overline{d \chi_{2}}}{d z_{R}}\right)\right\}+\{R \rightarrow L\}\right] \delta\left(p-p^{\prime}\right) \delta_{l l^{\prime}} \delta_{m m^{\prime}} \\
& =:\left(\left(\chi_{1}, \chi_{2}\right)\right) \delta\left(p-p^{\prime}\right) \delta_{l l^{\prime}} \delta_{m m^{\prime}}
\end{aligned}
$$

As will be discussed in Section IV, this is not always correct but we assume so for the time being.

Then the Klein-Gordon inner products of $\chi_{p}^{(R)}$ and $\chi_{p}^{(L)}$ are calculated to be

$$
\begin{aligned}
& \left(\left(\chi_{p}^{(R)}, \chi_{p}^{(L)}\right)\right)=\left(\left(\chi_{p}^{(L)}, \chi_{p}^{(R)}\right)\right)=0, \\
& \left(\left(\chi_{p}^{(R)}, \chi_{p}^{(R)}\right)\right)=\left(\left(\chi_{p}^{(L)}, \chi_{p}^{(L)}\right)\right)=\frac{2}{\pi} e^{-\pi p}\left(\cosh 2 \pi p-\cos 2 \pi \nu^{\prime}\right),
\end{aligned}
$$

where we have used the fact that

$$
\overline{P_{\nu^{\prime}}^{i p}}=P_{\nu^{\prime}}^{-i p}, \quad \overline{Q_{\nu^{\prime}}^{i p}}=e^{-2 \pi p} Q_{\nu^{\prime}}^{-i p},
$$

and the Wronskian relations among $P_{\nu^{\prime}}^{ \pm i p}$ and $Q_{\nu^{\prime}}^{ \pm i p}$ [23]. In the above and in the rest of this section, we assume $\nu^{\prime}$ to be real, i.e., $-1 / 2 \leq \nu^{\prime}<1(0 \leq \nu<3 / 2)$, in order to avoid inessential complexity. Extension to the case $\nu^{\prime}$ is imaginary, i.e., $\Re \nu^{\prime}=-1 / 2(\nu=$ pure imaginary) is straightforward. It then turns out that the following linear combinations of $\chi_{p}^{(R)}$ and $\chi_{p}^{(L)}$ are also mutually orthogonal:

$$
\begin{aligned}
& \chi_{p,+}=\frac{\chi_{p}^{(R)}+\chi_{p}^{(L)}}{\left(1+e^{-\pi p+\nu^{\prime} \pi i}\right) \Gamma\left(\nu^{\prime}+1+i p\right)} \\
& =\left\{\begin{array}{l}
\frac{1}{\Gamma\left(\nu^{\prime}+1+i p\right)}\left[P_{\nu^{\prime}}^{i p}\left(z_{R}\right)+\frac{i}{\pi}\left(1-e^{p \pi-i \nu^{\prime} \pi}\right) Q_{\nu^{\prime}}^{i p}\left(z_{R}\right)\right], \\
\frac{1}{\Gamma\left(\nu^{\prime}+1+i p\right)}\left[P_{\nu^{\prime}}^{i p}\left(z_{L}\right)+\frac{i}{\pi}\left(1-e^{p \pi-i \nu^{\prime} \pi}\right) Q_{\nu^{\prime}}^{i p}\left(z_{L}\right)\right],
\end{array}\right. \\
& \chi_{p,-}=\frac{\chi_{p}^{(R)}-\chi_{p}^{(L)}}{\left(1-e^{-\pi p+\nu^{\prime} \pi i}\right) \Gamma\left(\nu^{\prime}+1+i p\right)} \\
& =\left\{\begin{array}{l}
\frac{1}{\Gamma\left(\nu^{\prime}+1+i p\right)}\left[P_{\nu^{\prime}}^{i p}\left(z_{R}\right)+\frac{i}{\pi}\left(1+e^{p \pi-i \nu^{\prime} \pi}\right) Q_{\nu^{\prime}}^{i p}\left(z_{R}\right)\right], \\
-\frac{1}{\Gamma\left(\nu^{\prime}+1+i p\right)}\left[P_{\nu^{\prime}}^{i p}\left(z_{L}\right)+\frac{i}{\pi}\left(1+e^{p \pi-i \nu^{\prime} \pi}\right) Q_{\nu^{\prime}}^{i p}\left(z_{L}\right)\right] .
\end{array}\right.
\end{aligned}
$$

If we note the relation,

$$
Q_{\nu^{\prime}}^{i p}=\frac{\pi e^{-\pi p}}{2 i \sinh \pi p}\left[P_{\nu^{\prime}}^{i p}-\frac{\Gamma\left(\nu^{\prime}+1+i p\right)}{\Gamma\left(\nu^{\prime}+1-i p\right)} P_{\nu^{\prime}}^{-i p}\right]
$$

we may re-express $\chi_{p, \sigma}$ in terms of $P_{\nu^{\prime}}^{i p}$ alone as

$$
\chi_{p, \sigma}=\left\{\begin{array}{l}
\frac{1}{2 \sinh \pi p}\left(\frac{e^{\pi p}-\sigma e^{-i \pi \nu^{\prime}}}{\Gamma\left(\nu^{\prime}+i p+1\right)} P_{\nu^{\prime}}^{i p}\left(z_{R}\right)-\frac{e^{-\pi p}-\sigma e^{-i \pi \nu^{\prime}}}{\Gamma\left(\nu^{\prime}-i p+1\right)} P_{\nu^{\prime}}^{-i p}\left(z_{R}\right)\right), \\
\frac{\sigma}{2 \sinh \pi p}\left(\frac{e^{\pi p}-\sigma e^{-i \pi \nu^{\prime}}}{\Gamma\left(\nu^{\prime}+i p+1\right)} P_{\nu^{\prime}}^{i p}\left(z_{L}\right)-\frac{e^{-\pi p}-\sigma e^{-i \pi \nu^{\prime}}}{\Gamma\left(\nu^{\prime}-i p+1\right)} P_{\nu^{\prime}}^{-i p}\left(z_{L}\right)\right) .
\end{array}\right.
$$

Note that these mode functions have the symmetry $\chi_{-p, \sigma}=\chi_{p, \sigma}(\sigma= \pm)$, hence are even functions of $p$. Their Klein-Gordon norms are evaluated to give

$$
\left(\left(\chi_{p, \sigma}, \chi_{p, \sigma^{\prime}}\right)\right)=\frac{4\left(\cosh \pi p-\sigma \cos \nu^{\prime} \pi\right)}{\pi\left|\Gamma\left(\nu^{\prime}+1+i p\right)\right|^{2}} \delta_{\sigma \sigma^{\prime}}=: N_{p \sigma} \delta_{\sigma \sigma^{\prime}} .
$$


From these results, the field operator is now expanded as

$$
\hat{\phi}(x)=\int_{0}^{\infty} d p \sum_{\sigma, l, m}\left(\hat{a}_{p \sigma l m} v_{p \sigma l m}(x)+\hat{a}_{p \sigma l m}^{\dagger} \overline{v_{p \sigma l m}(x)}\right),
$$

where and in what follows, we use the symbol $v_{\Lambda}$ to denote the orthonormalized mode functions. In the present case, they are given by

$$
v_{p \sigma l m}(x)=\frac{1}{\sqrt{N_{p \sigma}}} \frac{\chi_{p, \sigma}(t)}{a(t)} f_{p l}(r) Y_{l m}(\Omega),
$$

with $(t, r)$ being either $\left(t_{R}, r_{R}\right)$ or $\left(t_{L}, r_{L}\right)$. In what follows, we suppress the subscript $R$ (or $\left.L\right)$ for notational simplicity unless ambiguity arises.

Then, for both $x^{\prime}$ and $x^{\prime \prime}$ in the region $R$ (or $L$ ), the Wightman function is given by

$$
\begin{aligned}
a\left(t^{\prime}\right) a\left(t^{\prime \prime}\right) G^{+}\left(x^{\prime}, x^{\prime \prime}\right) & =\int_{0}^{\infty} d p \sum_{\sigma, l, m} \frac{1}{N_{p \sigma}} \chi_{p, \sigma}\left(t^{\prime}\right) Y_{p l m}\left(r^{\prime}, \Omega^{\prime}\right) \overline{\chi_{p, \sigma}\left(t^{\prime \prime}\right) Y_{p l m}\left(r^{\prime \prime}, \Omega^{\prime \prime}\right)}, \\
& =\frac{1}{2 \pi^{2}} \int_{0}^{\infty} d p \frac{p \sin p \zeta}{\sinh \zeta} \sum_{\sigma= \pm} \frac{1}{N_{p \sigma}} \chi_{p, \sigma}\left(t^{\prime}\right) \overline{\chi_{p, \sigma}\left(t^{\prime \prime}\right)},
\end{aligned}
$$

where we have used the completeness relation for $Y_{p l m}$,

$$
\begin{aligned}
& \sum_{l, m} Y_{p l m}\left(r^{\prime}, \Omega^{\prime}\right) \overline{Y_{p l m}\left(r^{\prime \prime}, \Omega^{\prime \prime}\right)}=\frac{p \sin p \zeta}{2 \pi^{2} \sinh \zeta} \\
& \quad \cosh \zeta:=\cosh r^{\prime} \cosh r^{\prime \prime}-\sinh r^{\prime} \sinh r^{\prime \prime} \cos \Theta
\end{aligned}
$$

with $\cos \Theta$ being the directional cosine between $\Omega^{\prime}$ and $\Omega^{\prime \prime}$. Using the relations 2.18$)$ and $(2.20)$, and the symmetry $\chi_{-p, \sigma}=\chi_{p, \sigma}$ again, the above integral is written explicitly as

$$
\begin{aligned}
a\left(t^{\prime}\right) a\left(t^{\prime \prime}\right) G^{+}\left(x^{\prime}, x^{\prime \prime}\right)= & \frac{1}{8 \pi \sinh \zeta} \int_{-\infty}^{\infty} d p \frac{p \sin p \zeta}{\sinh \pi p} \\
\times & {\left[\frac{i e^{\pi\left(p-i \nu^{\prime}\right)}}{\sin \left(i p+\nu^{\prime}\right) \pi} P_{\nu^{\prime}}^{i p}\left(z^{\prime}\right) P_{\nu^{\prime}}^{-i p}\left(z^{\prime \prime}\right)-\frac{2 i \sin \nu^{\prime} \pi}{\pi \sin \left(i p+\nu^{\prime}\right) \pi} e^{-\pi p} P_{\nu^{\prime}}^{i p}\left(z^{\prime}\right) Q_{\nu^{\prime}}^{-i p}\left(z^{\prime \prime}\right)\right] } \\
= & \frac{1}{8 \pi \sinh \zeta} \frac{1}{2 \pi i} \int_{-\infty}^{\infty} i d p \frac{\pi p}{\sinh \pi p} e^{i p \zeta} \\
& \times\left[\frac{e^{\pi\left(p-i \nu^{\prime}\right)}}{\sin \left(i p+\nu^{\prime}\right) \pi} P_{\nu^{\prime}}^{i p}\left(z^{\prime}\right) P_{\nu^{\prime}}^{-i p}\left(z^{\prime \prime}\right)-\frac{2 \sin \nu^{\prime} \pi}{\pi \sin \left(i p+\nu^{\prime}\right) \pi} e^{-\pi p} P_{\nu^{\prime}}^{i p}\left(z^{\prime}\right) Q_{\nu^{\prime}}^{-i p}\left(z^{\prime \prime}\right)\right. \\
& \left.\quad+\frac{e^{-\pi\left(p+i \nu^{\prime}\right)}}{\sin \left(i p-\nu^{\prime}\right) \pi} P_{\nu^{\prime}}^{-i p}\left(z^{\prime}\right) P_{\nu^{\prime}}^{i p}\left(z^{\prime \prime}\right)-\frac{2 \sin \nu^{\prime} \pi}{\pi \sin \left(i p-\nu^{\prime}\right) \pi} e^{\pi p} P_{\nu^{\prime}}^{-i p}\left(z^{\prime}\right) Q_{\nu^{\prime}}^{i p}\left(z^{\prime \prime}\right)\right] .
\end{aligned}
$$

In deriving the above expression, we have assumed $\nu=\nu^{\prime}+1 / 2$ to be real. However, it can be shown that the final result (2.27) equally holds for imaginary $\nu$ without change. Note that this is true irrespective of the sign of $\nu$, i.e., for $\nu= \pm i|\nu|$. Note also that $p=0$ is no longer a pole for $\nu^{\prime} \neq$ integer, which we assume in the rest of this section. The cases $\nu^{\prime}=0(\nu=1 / 2$; massless conformal $)$ and $\nu^{\prime}=1(\nu=3 / 2$; massless minimal $)$ will be treated separately in Section V.

When $x^{\prime}$ and $x^{\prime \prime}$ are spatially separated, we may close the contour of integration over the upper half complex $p$-plain. Changing the integration variable to $u=i p$, Eq. (2.27) may be rewritten in the form,

$$
\begin{aligned}
a\left(t^{\prime}\right) a\left(t^{\prime \prime}\right) G^{+}\left(x^{\prime}, x^{\prime \prime}\right)= & \frac{1}{8 \pi \sinh \zeta} \frac{1}{2 \pi i} \int_{C} d u \frac{\pi u}{\sin \pi u} e^{-u \zeta} \\
& \left\{\frac{e^{i \pi\left(u-\nu^{\prime}\right)}}{\sin \pi\left(u-\nu^{\prime}\right)}\left(P_{\nu^{\prime}}^{-u}\left(z^{\prime}\right) P_{\nu^{\prime}}^{u}\left(z^{\prime \prime}\right)-\frac{2}{\pi} \sin \pi \nu^{\prime} e^{i \pi\left(\nu^{\prime}-2 u\right)} P_{\nu^{\prime}}^{-u}\left(z^{\prime}\right) Q_{\nu^{\prime}}^{u}\left(z^{\prime \prime}\right)\right)\right. \\
& \left.+\frac{e^{-i \pi\left(u+\nu^{\prime}\right)}}{\sin \pi\left(u+\nu^{\prime}\right)}\left(P_{\nu^{\prime}}^{u}\left(z^{\prime}\right) P_{\nu^{\prime}}^{-u}\left(z^{\prime \prime}\right)-\frac{2}{\pi} \sin \pi \nu^{\prime} e^{i \pi\left(\nu^{\prime}+2 u\right)} P_{\nu^{\prime}}^{u}\left(z^{\prime}\right) Q_{\nu^{\prime}}^{-u}\left(z^{\prime \prime}\right)\right)\right\},
\end{aligned}
$$


where the contour of integration $C$ on the complex $u$-plane is shown in Fig. 3. If we note the relations,

$$
P_{\nu^{\prime}}^{-n}\left(z^{\prime}\right) P_{\nu^{\prime}}^{n}\left(z^{\prime \prime}\right)=P_{\nu^{\prime}}^{n}\left(z^{\prime}\right) P_{\nu^{\prime}}^{-n}\left(z^{\prime \prime}\right), \quad P_{\nu^{\prime}}^{-n}\left(z^{\prime}\right) Q_{\nu^{\prime}}^{n}\left(z^{\prime \prime}\right)=P_{\nu^{\prime}}^{n}\left(z^{\prime}\right) Q_{\nu^{\prime}}^{-n}\left(z^{\prime \prime}\right),
$$

for integer $n$, we easily see that the residues of poles at $u=n(n=1,2,3, \cdots)$ cancel out completely. Thus the poles which contribute to the integral are classified into three classes.

$\operatorname{class}(a) ;$ poles of $1 / \sin \pi\left(u-\nu^{\prime}\right)$ :

$$
u=\nu^{\prime}+n, \quad\left\{\begin{array}{l}
n=1,2,3, \cdots,\left(-\frac{1}{2} \leq \nu^{\prime}<0, \Re \nu^{\prime}=-1 / 2\right) \\
n=0,1,2, \cdots,\left(0<\nu^{\prime}<1\right)
\end{array}\right.
$$

$\operatorname{class}(b) ;$ poles of $Q_{\nu^{\prime}}^{-u}\left(z^{\prime \prime}\right)$ :

$$
u=\nu^{\prime}+n, \quad n=1,2,3, \cdots
$$

$\operatorname{class}(c)$; poles of $1 / \sin \pi\left(u+\nu^{\prime}\right)$ :

$$
u=-\nu^{\prime}+n, \quad\left\{\begin{array}{l}
n=0,1,2, \cdots,\left(-\frac{1}{2} \leq \nu^{\prime}<0, \Re \nu^{\prime}=-1 / 2\right) \\
n=1,2,3, \cdots,\left(0<\nu^{\prime}<1\right)
\end{array}\right.
$$

The contribution from a class $(a)$ pole is given by

$$
a_{n}:=\frac{1}{8 \pi \sinh \zeta} \frac{(-1)^{n}}{\sin \pi \nu^{\prime}}\left(\nu^{\prime}+n\right) e^{-\left(\nu^{\prime}+n\right) \zeta}\left(P_{\nu^{\prime}}^{-\left(\nu^{\prime}+n\right)}\left(z^{\prime}\right) P_{\nu^{\prime}}^{\nu^{\prime}+n}\left(z^{\prime \prime}\right)-\frac{2}{\pi} \sin \pi \nu^{\prime} e^{-i \pi \nu^{\prime}} P_{\nu^{\prime}}^{-\left(\nu^{\prime}+n\right)}\left(z^{\prime}\right) Q_{\nu^{\prime}}^{\nu^{\prime}+n}\left(z^{\prime \prime}\right)\right) .
$$

By using the following formulae,

$$
\begin{aligned}
& Q_{\nu^{\prime}}^{\nu^{\prime}+n}(z)=\frac{\pi}{2} \frac{e^{i \pi \nu^{\prime}}}{\sin \pi \nu^{\prime}} P_{\nu^{\prime}}^{\nu^{\prime}+n}(z) ; \quad n=1,2,3, \cdots \\
& Q_{\nu^{\prime}}^{\nu^{\prime}-n}(z)=\frac{\pi}{2} \frac{e^{i \pi \nu^{\prime}}}{\sin \pi \nu^{\prime}}\left(P_{\nu^{\prime}}^{\nu^{\prime}-n}(z)-\frac{\Gamma\left(2 \nu^{\prime}-n+1\right)}{n !} P_{\nu^{\prime}}^{-\nu^{\prime}+n}(z)\right) ; \quad n=0,1,2, \cdots,
\end{aligned}
$$

we find

$$
\begin{aligned}
& a_{0}=\frac{1}{8 \pi \sinh \zeta} \frac{\nu^{\prime} \Gamma\left(2 \nu^{\prime}+1\right)}{\sin \pi \nu^{\prime}} e^{-\nu^{\prime} \zeta} P_{\nu^{\prime}}^{-\nu^{\prime}}\left(z^{\prime}\right) P_{\nu^{\prime}}^{-\nu^{\prime}}\left(z^{\prime \prime}\right), \\
& a_{n}=0 ; \quad n=1,2,3, \cdots .
\end{aligned}
$$

The contribution from a class (b) pole is given by

$$
b_{n}:=-\frac{1}{8 \pi \sinh \zeta} \frac{(-1)^{n} \pi}{\sin \pi \nu^{\prime}}\left(\nu^{\prime}+n\right) e^{-\left(\nu^{\prime}+n\right) \zeta} \frac{2}{\pi} \frac{e^{i \pi \nu^{\prime}} \sin \pi \nu^{\prime}}{\sin 2 \pi \nu^{\prime}} P_{\nu^{\prime}}^{\nu^{\prime}+n}\left(z^{\prime}\right)\left[\lim _{u \rightarrow \nu^{\prime}+n}\left(u-\nu^{\prime}-n\right) Q_{\nu^{\prime}}^{-u}\left(z^{\prime \prime}\right)\right] .
$$

The limit in the square bracket is evaluated as

$$
\begin{aligned}
\lim _{u \rightarrow \nu^{\prime}+n}\left(u-\nu^{\prime}-n\right) Q_{\nu^{\prime}}^{-u}\left(z^{\prime \prime}\right) & =\lim _{u \rightarrow \nu^{\prime}+n}\left(u-\left(\nu^{\prime}+n\right)\right) \Gamma\left(\nu^{\prime}-u+1\right) \frac{Q_{\nu^{\prime}}^{-u}\left(z^{\prime \prime}\right)}{\Gamma\left(\nu^{\prime}-u+1\right)} \\
& =\frac{(-1)^{n}}{(n-1) !} \frac{\pi}{2} \frac{e^{-i \pi \nu^{\prime}}}{\sin \pi \nu^{\prime} \Gamma\left(2 \nu^{\prime}+n+1\right)} P_{\nu^{\prime}}^{\nu^{\prime}+n}\left(z^{\prime \prime}\right) .
\end{aligned}
$$

Then we obtain

$$
b_{n}=-\frac{1}{8 \pi \sinh \zeta} \frac{\pi\left(\nu^{\prime}+n\right) e^{-\left(\nu^{\prime}+n\right) \zeta}}{(n-1) ! \sin \pi \nu^{\prime} \sin 2 \pi \nu^{\prime} \Gamma\left(2 \nu^{\prime}+n+1\right)} P_{\nu^{\prime}}^{\nu^{\prime}+n}\left(z^{\prime}\right) P_{\nu^{\prime}}^{\nu^{\prime}+n}\left(z^{\prime \prime}\right) ; \quad n=1,2,3, \cdots .
$$

The contribution from a class $(c)$ pole is given by

$$
c_{n}:=\frac{1}{8 \pi \sinh \zeta} \frac{(-1)^{n}}{\sin \pi \nu^{\prime}}\left(\nu^{\prime}-n\right) e^{\left(\nu^{\prime}-n\right) \zeta}\left(P_{\nu^{\prime}}^{-\nu^{\prime}+n}\left(z^{\prime}\right) P_{\nu^{\prime}}^{\nu^{\prime}-n}\left(z^{\prime \prime}\right)-\frac{2}{\pi} \sin \pi \nu^{\prime} e^{-i \pi \nu^{\prime}} P_{\nu^{\prime}}^{-\nu^{\prime}+n}\left(z^{\prime}\right) Q_{\nu^{\prime}}^{\nu^{\prime}-n}\left(z^{\prime \prime}\right)\right) .
$$


Using the second formula in Eq. (2.34), it reduces to

$$
c_{n}=\frac{1}{8 \pi \sinh \zeta} \frac{(-1)^{n}}{\sin \pi \nu^{\prime}}\left(\nu^{\prime}-n\right) e^{\left(\nu^{\prime}-n\right) \zeta} \frac{\Gamma\left(2 \nu^{\prime}-n+1\right)}{n !} P_{\nu^{\prime}}^{-\nu^{\prime}+n}\left(z^{\prime}\right) P_{\nu^{\prime}}^{-\nu^{\prime}+n}\left(z^{\prime \prime}\right) ; \quad n=0,1,2, \cdots .
$$

Combining these results, we obtain the Wightman function expressed in terms of the series sum of the residues. Here we focus on the case when $-1 / 2 \leq \nu^{\prime}<0$ or $\Re \nu^{\prime}=-1 / 2$. Then we have

$$
\begin{aligned}
G^{+}\left(x^{\prime}, x^{\prime \prime}\right) & =\frac{1}{8 \pi a\left(t^{\prime}\right) a\left(t^{\prime \prime}\right) \sinh \zeta \sin \pi \nu^{\prime}}\left\{-\frac{\pi}{\sin 2 \pi \nu^{\prime}} \sum_{n=1}^{\infty}\left(\nu^{\prime}+n\right) A_{n}+\sum_{n=0}^{\infty}\left(\nu^{\prime}-n\right) B_{n}\right\} \\
& =: G_{(A)}^{+}\left(x^{\prime}, x^{\prime \prime}\right)+G_{(B)}^{+}\left(x^{\prime}, x^{\prime \prime}\right),
\end{aligned}
$$

where

$$
\begin{aligned}
A_{n} & :=\frac{e^{-\left(\nu^{\prime}+n\right) \zeta}}{(n-1) ! \Gamma\left(2 \nu^{\prime}+n+1\right)} P_{\nu^{\prime}}^{\nu^{\prime}+n}\left(z^{\prime}\right) P_{\nu^{\prime}}^{\nu^{\prime}+n}\left(z^{\prime \prime}\right), \\
B_{n} & :=\frac{(-1)^{n} e^{\left(\nu^{\prime}-n\right) \zeta} \Gamma\left(2 \nu^{\prime}-n+1\right)}{n !} P_{\nu^{\prime}}^{-\nu^{\prime}+n}\left(z^{\prime}\right) P_{\nu^{\prime}}^{-\nu^{\prime}+n}\left(z^{\prime \prime}\right),
\end{aligned}
$$

and $G_{(A)}^{+}\left(x^{\prime}, x^{\prime \prime}\right)$ and $G_{(B)}^{+}\left(x^{\prime}, x^{\prime \prime}\right)$ correspond to the summations of $A_{n}$ and $B_{n}$ parts, respectively.

In the next section, we shall show that the above expression indeed coincides with the Euclidean vacuum Wightman function. The case $\nu^{\prime}>0$ will be discussed in Section IV.

\section{EUCLIDEAN VACUUM WIGHTMAN FUNCTION}

Equation (2.41) has been derived by assuming that the Klein-Gordon inner product can be evaluated in terms of the expression given by Eq. (2.17). If this assumption is valid, we expect Eq. (2.41) to coincide with the well-known Wightman function for the Euclidean vacuum [20],

$$
G_{E}^{+}\left(z^{\prime}, 0 ; z^{\prime \prime}, \zeta\right)=\frac{H^{2}}{8 \pi^{2}} \Gamma\left(\frac{3}{2}+\nu\right) \Gamma\left(\frac{3}{2}-\nu\right) \frac{P_{\nu^{\prime}}^{-1}(u)}{\sqrt{u^{2}-1}},
$$

where

$$
u:=s^{\prime} s^{\prime \prime} \cosh \zeta-z^{\prime} z^{\prime \prime} ; \quad s=\sqrt{z^{2}-1}=\sinh t .
$$

Now we prove that Eq. (2.41) is identical to the Euclidean vacuum Wightman function. For convenience, we denote the function given by the series expression (2.41) by $\tilde{G}^{+}\left(x^{\prime}, x^{\prime \prime}\right)$. The outline of the proof is as follows. First we show

that $\tilde{G}^{+}\left(x^{\prime}, x^{\prime \prime}\right)$ is de Sitter-invariant, i.e., it is a function of the invariant quantity $u$ alone. Then, since $\tilde{G}^{+}\left(x^{\prime}, x^{\prime \prime}\right)$ is guaranteed to be a solution of the field equation with respect to both of the arguments $x^{\prime}$ and $x^{\prime \prime}$ by construction, the form of $\tilde{G}^{+}\left(z^{\prime}, z^{\prime \prime}, \zeta\right)$ is restricted to be a linear combination of two independent solutions as

$$
\tilde{G}^{+}\left(z^{\prime}, z^{\prime \prime}, \zeta\right)=\frac{H^{2}}{8 \pi^{2}} \frac{\Gamma\left(\nu^{\prime}+2\right) \Gamma\left(-\nu^{\prime}+1\right)}{\sqrt{u^{2}-1}}\left(\alpha P_{\nu^{\prime}}^{-1}(u)+\beta \frac{\tan \pi \nu^{\prime}}{\pi} Q_{\nu^{\prime}}^{-1}(u)\right),
$$

where $\alpha$ and $\beta$ are some constants. Examining the asymptotic behaviour of the Wightman function for the limit of large spatial separation, $\alpha$ and $\beta$ are determined as $\{\alpha=1, \beta=0\}$, which is of the Euclidean vacuum Wightman function.

To show the de Sitter invariance of $\tilde{G}^{+}\left(z^{\prime}, z^{\prime \prime}, \zeta\right)$, we use the fact that if a function $F\left(z^{\prime}, z^{\prime \prime}, \zeta\right)$ satisfies the relations,

$$
\begin{aligned}
& \frac{\partial u}{\partial \zeta} \frac{\partial F\left(z^{\prime}, z^{\prime \prime}, \zeta\right)}{\partial z^{\prime}}=\frac{\partial u}{\partial z^{\prime}} \frac{\partial F\left(z^{\prime}, z^{\prime \prime}, \zeta\right)}{\partial \zeta}, \\
& \frac{\partial u}{\partial \zeta} \frac{\partial F\left(z^{\prime}, z^{\prime \prime}, \zeta\right)}{\partial z^{\prime \prime}}=\frac{\partial u}{\partial z^{\prime \prime}} \frac{\partial F\left(z^{\prime}, z^{\prime \prime}, \zeta\right)}{\partial \zeta},
\end{aligned}
$$

$F\left(z^{\prime}, z^{\prime \prime}, \zeta\right)$ is a function of $u$ only, i.e., $F\left(z^{\prime}, z^{\prime \prime}, \zeta\right)$ is a de Sitter-invariant function. Of course we can directly check the relations (3.4) and (3.5) for $F\left(z^{\prime}, z^{\prime \prime}, \zeta\right)=\tilde{G}^{+}\left(z^{\prime}, z^{\prime \prime}, \zeta\right)$, but it is easier to show these relations first for 


$$
F\left(z^{\prime}, z^{\prime \prime}, \zeta\right)=\frac{1}{\sin \pi \nu^{\prime}}\left(\frac{\pi}{\sin 2 \pi \nu^{\prime}} \sum_{n=1}^{\infty} A_{n}+\sum_{n=0}^{\infty} B_{n}\right) .
$$

Then if $F\left(z^{\prime}, z^{\prime \prime}, \zeta\right)$ is shown to be a function of $u$ alone, $\tilde{G}^{+}\left(z^{\prime}, z^{\prime \prime}, \zeta\right)$ can be obtained as

$$
8 \pi \tilde{G}^{+}\left(z^{\prime}, z^{\prime \prime}, \zeta\right)=\frac{1}{s^{\prime} s^{\prime \prime} \sinh \zeta} \frac{\partial}{\partial \zeta} F\left(u\left(z^{\prime}, z^{\prime \prime}, \zeta\right)\right)=\frac{d}{d u} F(u) .
$$

Hence $\tilde{G}^{+}\left(z^{\prime}, z^{\prime \prime}, \zeta\right)$ is also a function of only $u$ if $F\left(z^{\prime}, z^{\prime \prime}, \zeta\right)$ is so.

Now let us prove the relations (3.4) and (3.5). We define

$$
\begin{aligned}
& K_{n}^{(1)}:=\frac{\partial u}{\partial z^{\prime}} \frac{\partial A_{n}}{\partial \zeta}=-\left(\frac{s^{\prime \prime} z^{\prime}}{s^{\prime}} \cosh \zeta-z^{\prime \prime}\right)\left(\nu^{\prime}+n\right) P_{\nu^{\prime}}^{\nu^{\prime}+n}\left(z^{\prime}\right) \frac{e^{-\left(\nu^{\prime}+n\right) \zeta}}{(n-1) ! \Gamma\left(2 \nu^{\prime}+n+1\right)} P_{\nu^{\prime}}^{\nu^{\prime}+n}\left(z^{\prime \prime}\right), \\
& K_{n}^{(2)}:=\frac{\partial u}{\partial \zeta} \frac{\partial A_{n}}{\partial z^{\prime}}=\frac{s^{\prime \prime}}{s^{\prime}} \sinh \zeta\left(\left(\nu^{\prime}+n\right) z^{\prime} P_{\nu^{\prime}}^{\nu^{\prime}+n}\left(z^{\prime}\right)+s^{\prime} P_{\nu^{\prime}}^{\nu^{\prime}+n+1}\left(z^{\prime}\right)\right) \frac{e^{-\left(\nu^{\prime}+n\right) \zeta}}{(n-1) ! \Gamma\left(2 \nu^{\prime}+n+1\right)} P_{\nu^{\prime}}^{\nu^{\prime}+n}\left(z^{\prime \prime}\right), \\
& L_{n}^{(1)}:=\frac{\partial u}{\partial z^{\prime}} \frac{\partial B_{n}}{\partial \zeta}=\left(\frac{s^{\prime \prime} z^{\prime}}{s^{\prime}} \cosh \zeta-z^{\prime \prime}\right)\left(\nu^{\prime}-n\right) P_{\nu^{\prime}}^{-\nu^{\prime}+n}\left(z^{\prime}\right) \frac{(-1)^{n} e^{\left(\nu^{\prime}-n\right) \zeta} \Gamma\left(2 \nu^{\prime}-n+1\right)}{n !} P_{\nu^{\prime}}^{-\nu^{\prime}+n}\left(z^{\prime \prime}\right), \\
& L_{n}^{(2)}:=\frac{\partial u}{\partial \zeta} \frac{\partial B_{n}}{\partial z^{\prime}}=\frac{s^{\prime \prime}}{s^{\prime}} \sinh \zeta\left(-\left(\nu^{\prime}-n\right) z^{\prime} P_{\nu^{\prime}}^{-\nu^{\prime}+n}\left(z^{\prime}\right)+s^{\prime} P_{\nu^{\prime}}^{-\nu^{\prime}+n+1}\left(z^{\prime}\right)\right) \\
& \times \frac{(-1)^{n} e^{\left(\nu^{\prime}-n\right) \zeta} \Gamma\left(2 \nu^{\prime}-n+1\right)}{n !} P_{\nu^{\prime}}^{-\nu^{\prime}+n}\left(z^{\prime \prime}\right) .
\end{aligned}
$$

Then

$$
\begin{aligned}
2 \sum_{n=1}^{\infty}\left(K_{n}^{(1)}-K_{n}^{(2)}\right)=\sum_{n=1}^{\infty}[ & \left.\left(-2 \frac{s^{\prime \prime} z^{\prime}}{s^{\prime}} e^{\zeta}+2 z^{\prime \prime}\right)\left(\nu^{\prime}+n\right) P_{\nu^{\prime}}^{\nu^{\prime}+n}\left(z^{\prime}\right)-s^{\prime \prime}\left(e^{\zeta}-e^{-\zeta}\right) P_{\nu^{\prime}}^{\nu^{\prime}+n+1}\left(z^{\prime}\right)\right] \\
& \times \frac{e^{-\left(\nu^{\prime}+n\right) \zeta}}{(n-1) ! \Gamma\left(2 \nu^{\prime}+n+1\right)} P_{\nu^{\prime}+n}^{\nu^{\prime}+n}\left(z^{\prime \prime}\right) \\
=\sum_{n=0}^{\infty}[ & -\frac{1}{n ! \Gamma\left(2 \nu^{\prime}+n+2\right)}\left(2\left(\nu^{\prime}+n+1\right) \frac{z^{\prime}}{s^{\prime}} P_{\nu^{\prime}}^{\nu^{\prime}+n+1}\left(z^{\prime}\right)+P_{\nu^{\prime}}^{\nu^{\prime}+n+2}\left(z^{\prime}\right)\right) s^{\prime \prime} P_{\nu^{\prime}}^{\nu^{\prime}+n+1}\left(z^{\prime \prime}\right) \\
& +2 \frac{\nu^{\prime}+n}{(n-1) ! \Gamma\left(2 \nu^{\prime}+n+1\right)} P_{\nu^{\prime}+n}^{\nu^{\prime}+n}\left(z^{\prime}\right) z^{\prime \prime} P_{\nu^{\prime}}^{\nu^{\prime}+n}\left(z^{\prime \prime}\right) \\
& \left.+\frac{1}{(n-2) ! \Gamma\left(2 \nu^{\prime}+n\right)} P_{\nu^{\prime}+n}^{\nu^{\prime}}\left(z^{\prime}\right) s^{\prime \prime} P_{\nu^{\prime}}^{\nu^{\prime}+n-1}\left(z^{\prime \prime}\right)\right] e^{-\left(\nu^{\prime}+n\right) \zeta .}
\end{aligned}
$$

Each $n$ term in the above expression is shown to be zero by iteratively using the recursion formula,

$$
P_{\nu^{\prime}}^{\nu^{\prime}+n+2}(z)+2\left(\nu^{\prime}+n+1\right) \frac{z}{s} P_{\nu^{\prime}}^{\nu^{\prime}+n+1}(z)+n\left(2 \nu^{\prime}+n+1\right) P_{\nu^{\prime}}^{\nu^{\prime}+n}(z)=0 .
$$

In the same way, we have

$$
\begin{aligned}
2 \sum_{n=0}^{\infty}\left(L_{n}^{(1)}-L_{n}^{(2)}\right)=\sum_{n=0}^{\infty}[ & \left.\left(2 \frac{s^{\prime \prime} z^{\prime}}{s^{\prime}} e^{\zeta}-2 z^{\prime \prime}\right)\left(\nu^{\prime}-n\right) P_{\nu^{\prime}}^{-\nu^{\prime}+n}\left(z^{\prime}\right)-s^{\prime \prime}\left(e^{\zeta}-e^{-\zeta}\right) P_{\nu^{\prime}}^{-\nu^{\prime}+n+1}\left(z^{\prime}\right)\right] \\
& \times \frac{(-1)^{n} e^{\left(\nu^{\prime}-n\right) \zeta} \Gamma\left(2 \nu^{\prime}-n+1\right)}{n !} P_{\nu^{\prime}}^{-\nu^{\prime}+n}\left(z^{\prime \prime}\right) \\
=\sum_{n=-1}^{\infty}[ & -\frac{\Gamma\left(2 \nu^{\prime}-n\right)}{(n+1) !}\left(2\left(\nu^{\prime}-n-1\right) \frac{z^{\prime}}{s^{\prime}} P_{\nu^{\prime}}^{-\nu^{\prime}+n+1}\left(z^{\prime}\right)-P_{\nu^{\prime}}^{-\nu^{\prime}+n+2}\left(z^{\prime}\right)\right) s^{\prime \prime} P_{\nu^{\prime}}^{\nu^{\prime}+n+1}\left(z^{\prime \prime}\right) \\
& -2 \frac{\left(\nu^{\prime}-n\right) \Gamma\left(2 \nu^{\prime}-n+1\right)}{n !} P_{\nu^{\prime}}^{-\nu^{\prime}+n}\left(z^{\prime}\right) z^{\prime \prime} P_{\nu^{\prime}}^{-\nu^{\prime}+n}\left(z^{\prime \prime}\right) \\
& \left.-\frac{\Gamma\left(2 \nu^{\prime}-n+2\right)}{(n-1) !} P_{\nu^{\prime}}^{-\nu^{\prime}+n}\left(z^{\prime}\right) s^{\prime \prime} P_{\nu^{\prime}}^{-\nu^{\prime}+n-1}\left(z^{\prime \prime}\right)\right](-1)^{n} e^{\left(\nu^{\prime}-n\right) \zeta},
\end{aligned}
$$


and each $n$ term is shown to be zero by using the formula,

$$
P_{\nu^{\prime}}^{-\nu^{\prime}+n+2}(z)+2\left(-\nu^{\prime}+n+1\right) \frac{z}{s} P_{\nu^{\prime}}^{-\nu^{\prime}+n+1}(z)+(n+1)\left(-2 \nu^{\prime}+n\right) P_{\nu^{\prime}}^{-\nu^{\prime}+n}(z)=0 .
$$

Thus we find that $F\left(z^{\prime}, z^{\prime \prime}, \zeta\right)$ satisfies the condition (3.4). The condition (3.5) is also shown to be satisfied by replacing the roles of $z^{\prime}$ and $z^{\prime \prime}$. Hence we find that $\tilde{G}^{+}\left(z^{\prime}, z^{\prime \prime}, \zeta\right)$ is de Sitter-invariant as well as $F\left(z^{\prime}, z^{\prime \prime}, \zeta\right)$.

As mentioned before, since $\tilde{G}^{+}\left(z^{\prime}, z^{\prime \prime}, \zeta\right)$ is de Sitter-invariant, the fact that this function satisfies the field equation restricts its form to that given by Eq. (3.3). Moreover, as is apparent from the above proof of the de Sitter invariance, $G_{(A)}^{+}\left(z^{\prime}, z^{\prime \prime}, \zeta\right)$ and $G_{(B)}^{+}\left(z^{\prime}, z^{\prime \prime}, \zeta\right)$ are both de Sitter-invariant and satisfy the field equation independently. This means that these two functions give two different de Sitter-invariant functions. To determine $\alpha$ and $\beta$ for each of them, we examine their asymptotic behaviours at large $\zeta$ limit. In this limit, $2 u \rightarrow s^{\prime} s^{\prime \prime} e^{\zeta}$.

The asymptotic behaviours of $G_{(A)}^{+}\left(z^{\prime}, z^{\prime \prime}, \zeta\right)$ and $G_{(B)}^{+}\left(z^{\prime}, z^{\prime \prime}, \zeta\right)$ are determined by their leading terms. Noting that

$$
P_{\nu^{\prime}}^{\nu^{\prime}+1}(z) \rightarrow \frac{2^{\nu^{\prime}+1}}{\Gamma\left(-\nu^{\prime}\right)} s^{-\nu^{\prime}-1}, \quad P_{\nu^{\prime}}^{-\nu^{\prime}}(z) \rightarrow \frac{2^{-\nu^{\prime}}}{\Gamma\left(1+\nu^{\prime}\right)} s^{\nu^{\prime}},
$$

and

$$
\Gamma(2 w)=\frac{2^{2 w}}{2 \sqrt{\pi}} \Gamma(w) \Gamma\left(w+\frac{1}{2}\right)
$$

the asymptotic forms are explicitly written as

$$
\begin{aligned}
G_{(A)}^{+}\left(z^{\prime}, z^{\prime \prime}, \zeta\right) \rightarrow & -\frac{H^{2}}{4 \pi(2 u)} \frac{2 \pi^{3 / 2}\left(\nu^{\prime}+1\right)}{\sin \pi \nu^{\prime} \sin 2 \pi \nu^{\prime} \Gamma\left(\nu^{\prime}+1\right) \Gamma\left(\nu^{\prime}+3 / 2\right)\left[\Gamma\left(-\nu^{\prime}\right)\right]^{2}} e^{-\left(1+\nu^{\prime}\right) \zeta\left(s^{\prime} s^{\prime \prime}\right)^{-\left(\nu^{\prime}+1\right)}} \\
& =\frac{H^{2}}{4 \pi^{5 / 2}} \Gamma\left(-\nu^{\prime}-1 / 2\right) \Gamma\left(\nu^{\prime}+2\right)(2 u)^{-\nu^{\prime}-2} \\
G_{(B)}^{+}\left(z^{\prime}, z^{\prime \prime}, \zeta\right) \rightarrow & \frac{H^{2}}{4 \pi(2 u)} \frac{\nu^{\prime} \Gamma\left(\nu^{\prime}+1 / 2\right)}{\sqrt{\pi} \sin \pi \nu^{\prime} \Gamma\left(\nu^{\prime}+1\right)} e^{\nu^{\prime} \zeta}\left(s^{\prime} s^{\prime \prime}\right)^{\nu^{\prime}} \\
& =\frac{H^{2}}{4 \pi^{5 / 2}} \Gamma\left(\nu^{\prime}+1 / 2\right) \Gamma\left(-\nu^{\prime}+1\right)(2 u)^{\nu^{\prime}-1}
\end{aligned}
$$

Comparing these with the asymptotic form of the right hand side of Eq. (3.3),

$$
\begin{aligned}
& \frac{H^{2}}{8 \pi^{2}} \frac{\Gamma\left(\nu^{\prime}+2\right) \Gamma\left(-\nu^{\prime}+1\right)}{\sqrt{u^{2}-1}}\left(\alpha P_{\nu^{\prime}}^{-1}(u)+\frac{\beta}{\pi} \tan \pi \nu^{\prime} Q_{\nu^{\prime}}^{-1}(u)\right) \\
& \rightarrow \frac{H^{2}}{4 \pi^{5 / 2}}\left[\alpha \Gamma\left(\nu^{\prime}+1 / 2\right) \Gamma\left(-\nu^{\prime}+1\right)(2 u)^{\nu^{\prime}-1}+(\alpha+\beta) \Gamma\left(-\nu^{\prime}-1 / 2\right) \Gamma\left(\nu^{\prime}+2\right)(2 u)^{-\nu^{\prime}-2}\right],
\end{aligned}
$$

it is easy to see that $G_{(A)}^{+}\left(z^{\prime}, z^{\prime \prime}, \zeta\right)$ corresponds to the choice of the coefficients $\{\alpha=1, \beta=-1\}$, and $G_{(B)}^{+}\left(z^{\prime}, z^{\prime \prime}, \zeta\right)$ to $\{\alpha=0, \beta=1\}$. Thus the sum $\tilde{G}^{+}=G_{(A)}^{+}+G_{(B)}^{+}$correctly gives the Euclidean vacuum Wightman function. This completes our proof.

\section{EXTENSION TO $\nu^{\prime}>0$}

Although we have assumed $-1 / 2 \leq \nu^{\prime}<0$ or $\Re \nu^{\prime}=-1 / 2$ to derive the expression (2.41) for the Wightman function, if we examine the proof in the previous section that it coincides with the Euclidean vacuum Wightman function, we find it is valid irrespective of the value of $\nu^{\prime}$, that is $\tilde{G}^{+}=\tilde{G}_{E}^{+}$as long as the series converges. Then if we consider the case $\nu^{\prime}>0$, we find that $\tilde{G}^{+}\left(z^{\prime}, z^{\prime \prime}, \zeta\right)$ differs from the original $G^{+}\left(z^{\prime}, z^{\prime \prime}, \zeta\right)$, which is given by the integral of products of the mode functions over real values of $p$, Eq. (2.27), or the contour integral (2.28), because different poles contribute to the integral. Specifically the contribution of $a_{0}$ comes in and $c_{0}$ goes out as $\nu^{\prime}$ becomes positive. Thus

$$
\tilde{G}^{+}\left(z^{\prime}, z^{\prime \prime}, \zeta\right)-G^{+}\left(z^{\prime}, z^{\prime \prime}, \zeta\right)=-\frac{H^{2}}{s^{\prime} s^{\prime \prime}}\left(a_{0}-c_{0}\right)
$$




$$
\begin{gathered}
=-\frac{H^{2}}{8 \pi s^{\prime} s^{\prime \prime} \sinh \zeta}\left[\frac{\nu^{\prime} \Gamma\left(2 \nu^{\prime}+1\right)}{\sin \pi \nu^{\prime}} e^{-\nu^{\prime} \zeta} P_{\nu^{\prime}}^{-\nu^{\prime}}\left(z^{\prime}\right) P_{\nu^{\prime}}^{-\nu^{\prime}}\left(z^{\prime \prime}\right)\right. \\
\left.-\frac{\nu^{\prime} \Gamma\left(2 \nu^{\prime}+1\right)}{\sin \pi \nu^{\prime}} e^{\nu^{\prime} \zeta} P_{\nu^{\prime}}^{-\nu^{\prime}}\left(z^{\prime}\right) P_{\nu^{\prime}}^{-\nu^{\prime}}\left(z^{\prime \prime}\right)\right] \\
=\frac{H^{2}}{4 \pi^{5 / 2}} \Gamma\left(-\nu^{\prime}+1\right) \Gamma\left(\nu^{\prime}+1 / 2\right) \frac{\sinh \nu^{\prime} \zeta}{\sinh \zeta}\left(s^{\prime} s^{\prime \prime}\right)^{\nu^{\prime}-1} .
\end{gathered}
$$

Physical origin of this difference between $\nu^{\prime}<0$ and $\nu^{\prime}>0$ may be understood as follows. The inverse transformation of Eq. (2.25) is given by

$$
\sum_{\sigma= \pm} \chi_{p, \sigma}\left(z^{\prime}\right) \overline{\chi_{p, \sigma}\left(z^{\prime \prime}\right)}=\frac{4 \pi a\left(t^{\prime}\right) a\left(t^{\prime \prime}\right)}{p} \int_{0}^{\infty} \sinh ^{2} \zeta d \zeta \frac{\sin p \zeta}{\sinh \zeta} G^{+}\left(t^{\prime}, 0 ; t^{\prime \prime}, \zeta\right) .
$$

As seen from the asymptotic behaviour of the Euclidean vacuum Wightman function for large $\zeta$ given in Eq. (3.17) with $\{\alpha=1, \beta=0\}$, the integral in Eq. (4.2) becomes manifestly divergent for $\nu^{\prime}>0$ if we set $G^{+}=G_{E}^{+}$. This means that the Euclidean vacuum state cannot be described by any power spectrum in the usual sense in which the summation of products of the mode functions is taken over real values of $p$. In deriving the normalized set of mode functions in Section III, we did not assumed anything other than that the Klein-Gordon norm can be evaluated on two isolated hypersurfaces denoted by (I) and (II). Thus we conclude that the discrepancy at $\nu^{\prime}>0$ is due to the breakdown of this assumption.

Let us consider a linear functional space, $\mathcal{F}$, which consists of all normalizable functions which satisfy the field equation. Here the normalizable functions mean that they have finite Klein-Gordon norms, apart from the trivial factor of the $\delta$-function. The Klein-Gordon inner product is defined on a Cauchy surface and is independent of the choice of the surface. To evaluate it, there is one convenient choice of the hypersurface, (IV), which is specified by $r_{C}=$ constant and included in the region $C$ as shown in Fig. 2.

As the system has the $O(3,1)$ symmetry, it is natural to take the basis vectors of $\mathcal{F}$ from functions of the separated form as given in Eq. (2.13). Restricting $p^{2}$ to be positive, if we evaluate these mode functions on the hypersurface (IV), they diverge at $t_{C}=\pi / 2$ or $t_{C}=-\pi / 2$ with infinite cycles of oscillation. However the oscillatory divergence is not so seriously bad that the Klein-Gordon norm still has a finite value. It then follows that two modes which have different separation constants $p^{2}$ and ${p^{\prime}}^{2}$ are orthogonal. Therefore they are contained in $\mathcal{F}$ and may be chosen to be the basis vectors. Moreover, as the mode function $f_{p l}\left(r_{C}\right)$ decreases rapidly as $r_{C}$ increases, after the deformation of the hypersurface (IV) to that consists of (I), (II) and (III), the contribution to the Klein-Gordon norm from (III) can be neglected as was assumed before. Thus the Klein-Gordon norm is correctly given by the expression (2.16). For completeness, in Appendix A we show that the Klein-Gordon inner products evaluated on the hypersurface (IV) are finite for mode functions with $p^{2}>0$ and in fact coincide with those evaluated on (I) and (II).

On the other hand, for $p^{2}<0$, almost all modes diverge at $t_{C}=\pi / 2$ or $t_{C}=-\pi / 2$ faster than $(t \mp \pi / 2)^{-1}$ (the critical power exponent depends on the dimension) and their Klein-Gordon norms diverge. Hence they are not contained in $\mathcal{F}$. However, when $\nu^{\prime}>0$, there exists an exceptionally set of modes with $p=i \nu^{\prime}$ which behave well enough,

$$
u_{(*) l m}=H\left(-i \cos t_{C}\right)^{\nu^{\prime}-1} \frac{P_{-\nu^{\prime}-1 / 2}^{-l-1 / 2}\left(i \sinh r_{C}\right)}{\sqrt{i \cosh r_{C}}} Y_{l m}(\Omega) .
$$

Its Klein-Gordon norm is finite and is given by

$$
\begin{aligned}
\left\langle u_{(*) l m}, u_{(*) l^{\prime} m^{\prime}}\right\rangle & =\frac{i \cosh ^{2} r_{C}}{H^{2}} \int_{-\pi / 2}^{\pi / 2} d t_{C} \cos t_{C} \int d \Omega\left\{\frac{\partial u_{(*) l m}}{\partial r_{C}} \overline{u_{(*) l^{\prime} m^{\prime}}}-u_{(*) l m} \frac{\partial \overline{u_{(*) l^{\prime} m^{\prime}}}}{\partial r_{C}}\right\} \\
& =\frac{2 \sqrt{\pi} \Gamma\left(\nu^{\prime}\right)}{\Gamma\left(\nu^{\prime}+1 / 2\right) \Gamma\left(-\nu^{\prime}+l+1\right) \Gamma\left(\nu^{\prime}+l+1\right)} \delta_{l l^{\prime}} \delta_{m m^{\prime}} \\
& =: N_{(*) l} \delta_{l l^{\prime}} \delta_{m m^{\prime}} .
\end{aligned}
$$

In the region $R$, these mode functions take the form,

$$
u_{(*) l m}(x)=H\left(\sinh t_{R}\right)^{\nu^{\prime}-1} \frac{P_{-\nu^{\prime}-1 / 2}^{-l-1 / 2}\left(\cosh r_{R}\right)}{\sqrt{\sinh r_{R}}} Y_{l m}(\Omega) .
$$


They have exactly the same functional form in the region $L$ as well, with $\left(t_{R}, r_{R}\right)$ replaced by $\left(t_{L}, r_{L}\right)$. Therefore the contribution to the Wightman function from these modes in the region $R$ or $L$ becomes (with the suffix $R$ or $L$ suppressed)

$$
\begin{aligned}
G_{(*)}^{+}\left(z^{\prime}, z^{\prime \prime}, \zeta\right):= & \sum_{l, m} \frac{u_{(*) l m} \overline{u_{(*) l m}}}{N_{(*) l}} \\
= & \frac{\Gamma\left(\nu^{\prime}+1 / 2\right)}{2 \sqrt{\pi} \Gamma\left(\nu^{\prime}\right)} \sum_{l, m} \Gamma\left(-\nu^{\prime}+l+1\right) \Gamma\left(\nu^{\prime}+l+1\right) \\
& \times H^{2}\left(s^{\prime} s^{\prime \prime}\right) \nu^{\prime}-1 \frac{P_{-\nu^{\prime}-1 / 2}^{-l-1 / 2}\left(\cosh r^{\prime}\right)}{\sqrt{\sinh r^{\prime}}} \frac{P_{-\nu^{\prime}-1 / 2}^{-l-1 / 2}\left(\cosh r^{\prime \prime}\right)}{\sqrt{\sinh r^{\prime \prime}}} Y_{l} m\left(\Omega^{\prime}\right) \overline{Y_{l} m\left(\Omega^{\prime \prime}\right)} \\
= & \frac{H^{2}}{8 \pi^{3 / 2}} \Gamma\left(-\nu^{\prime}+1\right) \Gamma\left(\nu^{\prime}+1 / 2\right) \nu^{\prime} \frac{P_{-\nu^{\prime}-1 / 2}^{-1 / 2}(\cosh \zeta)}{\sqrt{\sinh \zeta}}\left[\lim _{r \rightarrow 0} \frac{P_{-\nu^{\prime}-1 / 2}^{-1 / 2}(\cosh r)}{\sqrt{\sinh r}}\right]\left(s^{\prime} s^{\prime \prime}\right) \nu^{\prime}-1 \\
= & \frac{H^{2}}{4 \pi^{5 / 2}} \Gamma\left(-\nu^{\prime}+1\right) \Gamma\left(\nu^{\prime}+1 / 2\right) \frac{\sinh \nu^{\prime} \zeta}{\sinh \zeta}\left(s^{\prime} s^{\prime \prime}\right)^{\nu^{\prime}-1},
\end{aligned}
$$

where, in the third equality, the relation required by the $O(3,1)$ invariance,

$$
\begin{aligned}
\sum_{l, m} 4 \pi \frac{\Gamma\left(-\nu^{\prime}+l+1\right) \Gamma\left(\nu^{\prime}+l+1\right)}{\Gamma\left(-\nu^{\prime}+1\right) \Gamma\left(\nu^{\prime}+1\right)} & \frac{P_{-\nu^{\prime}-1 / 2}^{-l-1 / 2}\left(\cosh r^{\prime}\right)}{\sqrt{\sinh r^{\prime}}} \frac{P_{-\nu^{\prime}-1 / 2}^{-l-1 / 2}\left(\cosh r^{\prime \prime}\right)}{\sqrt{\sinh r^{\prime \prime}}} Y_{l} m\left(\Omega^{\prime}\right) \overline{Y_{l} m\left(\Omega^{\prime \prime}\right)} \\
& =\frac{P_{-\nu^{\prime}-1 / 2}^{-1 / 2}(\cosh \zeta)}{\sqrt{\sinh \zeta}} \lim _{r \rightarrow 0}\left[\frac{P_{-\nu^{\prime}-1 / 2}^{-1 / 2}(\cosh r)}{\sqrt{\sinh r}}\right] \\
& =\sqrt{\frac{2}{\pi} \frac{P_{-\nu^{\prime}-1 / 2}^{-1 / 2}(\cosh \zeta)}{\sqrt{\sinh \zeta}}},
\end{aligned}
$$

has been used. For $\nu^{\prime}=-i p(p>0)$, this is just the equation describing the completeness of $Y_{p l m}$, Eq. (2.26), which is well-known to hold. In Appendix B, we show it holds for $\nu^{\prime}>0, \nu^{\prime} \neq$ integer as well. Thus we find that

$$
\tilde{G}^{+}\left(z^{\prime}, z^{\prime \prime}, \zeta\right)=G^{+}\left(z^{\prime}, z^{\prime \prime}, \zeta\right)+G_{(*)}^{+}\left(z^{\prime}, z^{\prime \prime}, \zeta\right)
$$

As clear from the above, the degrees of freedom described by $u_{(*) l m}$ cannot be quantized in the open chart, where these modes are not normalizable. However if the quantization is performed on some other hypersurfaces, say, the time constant hypersurfaces of a closed chart, we do not confront with any problem in the quantization. We may then quantize the field there, but instead of using the harmonics on the three-sphere, we may expand the field operator in terms of the mode functions associated with an open chart,

$$
\hat{\phi}(x)=\int_{0}^{\infty} d p \sum_{\sigma, l, m} v_{p \sigma l m}(x) \hat{a}_{p \sigma l m}+\sum_{l, m} v_{(*) l m} \hat{a}_{(*) l m}+h . c . ; \quad 1>\nu^{\prime}>0,
$$

where the orthonormalized mode functions are denoted by $v_{\Lambda}$ as before (see Eq. (2.24) for $\nu^{\prime}<0$ ) and are now given by

$$
\begin{aligned}
& v_{p \sigma l m}(x)=\frac{H}{\sqrt{N_{p \sigma}}} \frac{\chi_{p, \sigma}(t)}{\sinh t} f_{p l}(r) Y_{l m}(\Omega), \\
& v_{(*) l m}(x)=\frac{H}{\sqrt{N_{(*) l}}}(\sinh t)^{\nu^{\prime}-1} \frac{P_{-\nu^{\prime}-1 / 2}^{-l-1 / 2}(\cosh r)}{\sqrt{\sinh r}} Y_{l m}(\Omega) .
\end{aligned}
$$

In the above, the coordinates $(t, r)$ stand for $\left(t_{R}, r_{R}\right)$ or $\left(t_{L}, r_{L}\right)$ if $x$ is in the region $R$ or $L$, respectively, and for $\left.\left(i\left( \pm t_{C}-\pi / 2\right), r_{C}+i \pi / 2\right)\right)$ in the region $C$ where the upper (lower) sign corresponds to the analytic continuation from the region $R(L)$ as specified in Eq. (2.3).

Thus we have found a complete prescription to express the Euclidean vacuum state in terms of the mode functions associated with an open chart. Even in general situations in which the background differs from the exact de Sitter 
space and the mass of the scalar field changes in time, the above method of decomposing the field operator in terms of the mode functions which respect the $O(3,1)$ symmetry will be a very useful tool to evaluate the evolution of the field as long as the quantum state is initially in the Euclidean vacuum. Furthermore, we expect that the logical procedure given here should play an important role when we consider various problems associated with the quantum tunneling through $O(4)$-symmetric bubble nucleation.

\section{CONFORMAL AND MINIMAL MASSLESS CASE}

In this section we consider the cases $\nu^{\prime}=0$ and $\nu^{\prime} \rightarrow 1$, which correspond to the conformal massless case and the minimal massless limit, respectively.

\section{A. Massless conformal coupling case}

First we consider the case $\nu^{\prime}=0$. In this case the integration of Eqs. (2.25) and (4.2) can be easily performed because the Legendre function reduces to an elementary function as

$$
P_{0}^{i p}(\cosh t)=\frac{e^{-i p \eta}}{i p \Gamma(-i p)}
$$

where $\eta$ is related to $t$ by the relation, $\sinh \eta=-1 / \sinh t$. Then, the right hand side of Eq. (2.25) may be expressed as

$$
\frac{1}{4 \pi^{2}} \int_{0}^{\infty} d p \frac{\sin p \zeta}{\sinh \zeta}\left\{\frac{1}{1-e^{-2 \pi p}} e^{-i p\left(\eta^{\prime}-\eta^{\prime \prime}\right)}+\frac{e^{-2 \pi p}}{1-e^{-2 \pi p}} e^{i p\left(\eta^{\prime}-\eta^{\prime \prime}\right)}\right\}
$$

This integral can be easily done by closing the contour and evaluating its residues. Then we find that it coincides with the Euclidean vacuum Wightman function,

$$
G^{+}\left(z^{\prime}, z^{\prime \prime}, \zeta\right)=\frac{H^{2}}{8 \pi^{2}} \frac{1}{1+u} .
$$

If we focus on either of the region $R$ or $L$, a natural choice of the positive frequency function is $\tilde{\chi}_{p}=e^{-i p \eta} / \sqrt{2 p}$. Interpreting Eq. (5.2) in terms of these mode functions, the Euclidean vacuum is seen as a thermal state, as was pointed out in [21], This thermal nature may be understood as a consequence of the loss of information. For complete description of a quantum state, it is necessary to specify the states both in the regions $R$ and $L$. Hence if we consider the expectation value of an observable which has support only in the region $R$ (or $L$ ), it does not depend on the quantum state of the region $L$ ( or $R$ ). This effectively brings a pure state into a mixed state. Of course, this phenomenon is not peculiar only for $\nu^{\prime}=0$ but it appears in the easiest form for this special case. In fact, the expression for $\chi_{p \sigma}$ given in Eq.(2.21) does already indicate the thermal nature; the ratio of the squared absolute values

of the coefficients in front of $P_{\nu^{\prime}}^{i p}$ ("positive frequency" in the natural sense) and $P_{\nu^{\prime}}^{-i p}$ ("negative frequency"), after normalized by $N_{p \sigma}$ and summed over $\sigma= \pm$, is $e^{2 \pi p}$.

\section{B. Massless minimal coupling limit}

Next let us consider the massless minimal coupling limit, $\nu^{\prime} \rightarrow 1$. Using the following formulae,

$$
\begin{aligned}
P_{1+\epsilon}^{-1}(u) & =\left(\frac{u-1}{u+1}\right)^{1 / 2}\left\{\frac{u+1}{2}+\frac{\epsilon}{2}\left(\frac{u-1}{2}+(u+1) \ln \left(\frac{u+1}{2}\right)\right)\right\}+O\left(\epsilon^{2}\right), \\
\Gamma(3+\epsilon) & =2+\epsilon(3-2 \gamma)+O\left(\epsilon^{2}\right), \\
\Gamma(-\epsilon) & =-\frac{1}{\epsilon}-\gamma+O(\epsilon),
\end{aligned}
$$

for $\epsilon \rightarrow 0$, the $\nu^{\prime} \rightarrow 1$ limit of $G_{E}^{+}\left(z^{\prime}, z^{\prime \prime}, \zeta\right)$ is given by 


$$
G_{E}^{+}\left(z^{\prime}, z^{\prime \prime}, \zeta\right) \rightarrow \frac{H^{2}}{8 \pi^{2}}\left\{-\frac{1}{\nu^{\prime}-1}+\left[\frac{1}{u+1}-\ln \left(\frac{u+1}{2}\right)-2\right]+O\left(\nu^{\prime}-1\right)\right\} .
$$

In the same limit, the contribution from $p=i \nu^{\prime}$ modes to the Wightman function given by Eq. (4.6) becomes

$$
G_{(*)}^{+}\left(z^{\prime}, z^{\prime \prime}, \zeta\right) \rightarrow-\frac{H^{2}}{8 \pi^{2}}\left\{\frac{1}{\nu^{\prime}-1}+\left[\zeta \operatorname{coth} \zeta+\ln \left(\frac{s^{\prime} s^{\prime \prime}}{4}\right)+2\right]+O\left(\nu^{\prime}-1\right)\right\} .
$$

On the other hand, for $\nu^{\prime}=1$, Eq. (2.25) reduces to a very simple form,

$$
a\left(\eta^{\prime}\right) a\left(\eta^{\prime \prime}\right) G^{+}\left(z^{\prime}, z^{\prime \prime}, \zeta\right)=\frac{1}{8 \pi^{2}} \int_{-\infty}^{\infty} d p \frac{\sin p \zeta}{\sinh \zeta} \frac{e^{\pi p}}{\sinh \pi p} \frac{\left(z^{\prime}+i p\right)\left(z^{\prime \prime}-i p\right)}{1+p^{2}} e^{-i p\left(\eta^{\prime}-\eta^{\prime \prime}\right)} .
$$

The above integration can be done elementarily to give

$$
G^{+}\left(z^{\prime}, z^{\prime \prime}, \zeta\right)=\frac{H^{2}}{8 \pi^{2}}\left\{\frac{1}{1+u}-\ln \left(\frac{1+u}{2}\right)+\ln \left(\frac{s^{\prime} s^{\prime \prime}}{4}\right)+\zeta \operatorname{coth} \zeta\right\} .
$$

Adding Eqs. (5.6) and (5.8), we immediately see that $\tilde{G}^{+}\left(z^{\prime}, z^{\prime \prime}, \zeta\right)=G_{E}^{+}\left(z^{\prime}, z^{\prime \prime}, \zeta\right)$. We note that the Wightman function same as Eq. (5.8) has been obtained by Allen and Caldwell [9] in a totally different fashion.

\section{CONCLUSION}

We have derived the expression for the Euclidean vacuum Wightman function of a scalar field in de Sitter space in terms of the mode functions associated with the harmonic functions on an open chart, $Y_{p l m}$, i.e., on hyperbolic time slices which respect the $O(3,1)$ symmetry. The formula we have obtained is applicable to any mass or curvature coupling, provided the effective mass square is positive, i.e., $M_{e f f}^{2}=M^{2}+12 \xi H^{2}>0$. Usually the power spectrum of the quantum fluctuations is described by the modes with real values of comoving wavenumber $p$. However we have found that the Euclidean vacuum Wightman function cannot be described in this standard manner for $M_{\text {eff }}^{2}<2 H^{2}$. We have found that an additional contribution from the modes with an imaginary value of $p$ is necessary to describe the Euclidean vacuum Wightman function in this case. These modes are not square-integrable on a hyperbolic time slice in an open chart but their Klein-Gordon norms are finite if we take a complete Cauchy surface in de Sitter space to evaluate them.

The expressions for the Euclidean vacuum Wightman function and the field operator we have obtained will be a powerful tool to calculate observable quantities in open universe inflation models such as the cosmic temperature fluctuations when the initial quantum state is in the Euclidean vacuum $[8$. 10 . Furthermore, the technique developed in this paper to obtain a set of orthonormalized mode functions associated with an open chart should be applicable to more general situations provided the system has the $O(3,1)$ symmetry. The issue of the quantum state after false vacuum decay through $O(4)$-symmetric bubble nucleation is one interesting example of such situations. This issue has been discussed and investigated by several authors 13 17 but in a rather formal manner. In particular, although a method to determine the unnormalized mode functions after tunneling has been given, the normalization procedure applicable to general situations has not been explicitly given, except for the case of flat spacetime background [18]. Application of our normalization technique to some specific models of false vacuum decay will be discussed in a forthcoming paper.

\section{ACKNOWLEDGMENTS}

We would like to thank B. Allen, R. Caldwell, T. Hamazaki, D. Lyth, and N. Turok for enlightening discussions. One of us (M.S.) would like to thank for the hospitality given to him by the people at the Newton Institute, Cambridge University, where a part of this work was done. This work was supported in part by Monbusho Grant-in-Aid for Scientific Research Nos. 2010, 2841 and 05640342 and the Sumitomo Foundation. 


\section{APPENDIX A:}

Here we show the Klein-Gordon inner products on the hypersurface (IV) are finite for the mode functions with $p^{2}>0$ and they coincides with those evaluated on the hypersurfaces (I) and (II).

We consider the mode function $u_{p \sigma l m}$ given by

$$
u_{p \sigma l m}(x)=\frac{\chi_{p, \sigma}\left(t_{C}\right)}{a\left(t_{C}\right)} f_{p l}\left(r_{C}\right) Y_{l m}(\Omega),
$$

where $a\left(t_{C}\right)=H^{-1} \cos t_{C}, \chi_{p, \sigma}$ is given by Eq. (2.19) and $f_{p l}$ is by Eq. (2.11), both with relevant analytic continuations to the region $C$ specified in Eq. (2.3). In particular, the analytic continuation of $f_{p l}$ to the region $C$ is given by

$$
f_{p l}\left(r_{C}\right)=\frac{\Gamma(i p+l+1)}{\Gamma(i p)} \frac{P_{i p-1 / 2}^{-l-1 / 2}\left(i \sinh r_{C}\right)}{\sqrt{i \cosh r_{C}}},
$$

which plays the role of the positive frequency function. The Klein-Gordon inner products among $u_{p \sigma l m}$ on the $r_{C}=$ constant hypersurface (IV) take the form,

$$
\begin{aligned}
N_{p \sigma l m, p^{\prime} \sigma^{\prime} l^{\prime} m^{\prime}} & :=\left\langle u_{p \sigma l m}, u_{p^{\prime} \sigma^{\prime} l^{\prime} m^{\prime}}\right\rangle \\
& =\frac{i \cosh ^{2} r_{C}}{H^{2}} \int_{-\pi / 2}^{\pi / 2} d t_{C} \cos t_{C} \int d \Omega\left\{\frac{\partial u_{p \sigma l m}}{\partial r_{C}} \overline{u_{p^{\prime} \sigma^{\prime} l^{\prime} m^{\prime}}}-u_{p \sigma l m} \frac{\partial \overline{u_{p^{\prime} \sigma^{\prime} l^{\prime} m^{\prime}}}}{\partial r_{C}}\right\} \\
& =N_{p l}^{(1)} N_{p \sigma, p^{\prime} \sigma^{\prime}}^{(2)} \delta_{l l^{\prime}} \delta_{m m^{\prime}}
\end{aligned}
$$

where

$$
N_{p l}^{(1)}:=i \cosh ^{2} r_{C}\left\{\frac{\partial f_{p l}}{\partial r_{C}} \overline{f_{p l}}-f_{p l} \frac{\partial \overline{f_{p l}}}{\partial r_{C}}\right\}, \quad N_{p \sigma, p^{\prime} \sigma^{\prime}}^{(2)}:=\int_{-\pi / 2}^{\pi / 2} \frac{d t_{C}}{\cos t_{C}} \chi_{p, \sigma} \overline{\chi_{p^{\prime}, \sigma^{\prime}}}
$$

In the above, we have anticipated that the factor $N_{p \sigma, p^{\prime} \sigma^{\prime}}^{(2)}$ should contain the delta function $\delta\left(p-p^{\prime}\right)$ so that we may put $p^{\prime}=p$ when evaluating $N_{p l}^{(1)}$. This will be justified shortly.

The evaluation of the factor $N_{p l}^{(1)}$ is straightforward. We immediately obtain

$$
N_{p l}^{(1)}=\frac{2 p}{\pi} \sinh \pi p
$$

The evaluation of the factor $N_{p \sigma, p^{\prime} \sigma^{\prime}}^{(2)}$ needs a bit of consideration. First, from the symmetry that $\chi_{p, \sigma}\left(t_{C}\right)=\chi_{p, \sigma}\left(\sigma t_{C}\right)$ $(\sigma= \pm)$, we see that $N_{p \sigma, p^{\prime} \sigma^{\prime}}^{(2)}$ must be proportional to $\delta_{\sigma \sigma^{\prime}}$. Next, by using the field equation for $\chi_{p, \sigma}$,

$$
-\left[\frac{\partial}{\partial t_{C}} \cos ^{3} r_{C} \frac{\partial}{\partial t_{C}} \frac{1}{\cos r_{C}}+\left(\nu^{2}-\frac{9}{4}\right) \cos ^{2} r_{C}\right] \chi_{p, \sigma}=\left(1+p^{2}\right) \chi_{p, \sigma},
$$

we find

$$
\left(1+p^{2}\right) \int_{-\pi / 2}^{\pi / 2} d t_{C}\left(\cos t_{C}\right)^{-1} \chi_{p, \sigma} \overline{\chi_{p^{\prime}, \sigma^{\prime}}}=\left(1+{p^{\prime}}^{2}\right) \int_{-\pi / 2}^{\pi / 2} d t_{C}\left(\cos t_{C}\right)^{-1} \chi_{p, \sigma} \overline{\chi_{p^{\prime}, \sigma^{\prime}}}
$$

which implies that $N_{p \sigma, p^{\prime} \sigma^{\prime}}^{(2)}=0$ if ${p^{\prime}}^{2} \neq p^{2}$. Hence it should be expressed as

$$
N_{p \sigma, p^{\prime} \sigma^{\prime}}^{(2)}=N_{p \sigma}^{(2)} \delta\left(p-p^{\prime}\right) \delta_{\sigma \sigma^{\prime}},
$$

where we have restricted $p$ and $p^{\prime}$ to be positive (negative $p$ modes are equivalent to positive ones because of the symmetry $\chi_{-p, \sigma}=\chi_{p, \sigma}$ ). Thus our remaining task is to evaluate $N_{p \sigma}^{(2)}$.

It turns out that $N_{p \sigma}^{(2)}$ for real $p$ can be evaluated without detailed knowledge of the behavior of $\chi_{p, \sigma}$ because the divergent contribution at $p^{\prime}=p$ which determines the coefficient of the $\delta$-function comes only from the boundaries of 
integration at $t_{C}= \pm \pi / 2$. Therefore, to obtain $N_{p \sigma}^{(2)}$, we only have to know the behaviour of $\chi_{p, \sigma}$ near the boundaries. For this purpose, we use the expression (2.21) for $\chi_{p, \sigma}$, which we recapitulate:

$$
\chi_{p, \sigma}=\left\{\begin{array}{l}
\frac{1}{2 \sinh \pi p}\left(\frac{e^{\pi p}-\sigma e^{-i \pi \nu^{\prime}}}{\Gamma\left(\nu^{\prime}+i p+1\right)} P_{\nu^{\prime}}^{i p}\left(z_{R}\right)-\frac{e^{-\pi p}-\sigma e^{-i \pi \nu^{\prime}}}{\Gamma\left(\nu^{\prime}-i p+1\right)} P_{\nu^{\prime}}^{-i p}\left(z_{R}\right)\right), \\
\frac{\sigma}{2 \sinh \pi p}\left(\frac{e^{\pi p}-\sigma e^{-i \pi \nu^{\prime}}}{\Gamma\left(\nu^{\prime}+i p+1\right)} P_{\nu^{\prime}}^{i p}\left(z_{L}\right)-\frac{e^{-\pi p}-\sigma e^{-i \pi \nu^{\prime}}}{\Gamma\left(\nu^{\prime}-i p+1\right)} P_{\nu^{\prime}}^{-i p}\left(z_{L}\right)\right) .
\end{array}\right.
$$

Then noting the asymptotic behavior of $P_{\nu^{\prime}}^{i p}$ near the boundaries,

$$
P_{\nu^{\prime}}^{i p}\left(z_{R}\right) \sim \frac{2^{i p} t_{R}^{-i p}}{\Gamma(1-i p)}=\frac{2^{i p} e^{-\pi p / 2}\left(-t_{C}+\pi / 2\right)^{-i p}}{\Gamma(1-i p)}, \quad P_{\nu^{\prime}}^{i p}\left(z_{L}\right) \sim \frac{2^{i p} t_{L}^{-i p}}{\Gamma(1-i p)}=\frac{2^{i p} e^{-\pi p / 2}\left(t_{C}+\pi / 2\right)^{-i p}}{\Gamma(1-i p)},
$$

we find

$$
\chi_{p, \sigma} \sim \begin{cases}\alpha_{p}\left(-t_{C}+\pi / 2\right)^{-i p}-\beta_{p}\left(-t_{C}+\pi / 2\right)^{i p} & \text { for } \quad t_{C} \rightarrow \pi / 2-0, \\ \sigma\left(\alpha_{p}\left(t_{C}+\pi / 2\right)^{-i p}-\beta_{p}\left(t_{C}+\pi / 2\right)^{i p}\right) & \text { for } \quad t_{C} \rightarrow-\pi / 2+0,\end{cases}
$$

where

$$
\begin{aligned}
& \alpha_{p}=\frac{e^{-\pi p / 2}\left(e^{\pi p}-\sigma e^{-i \pi \nu^{\prime}}\right)}{2 \sinh \pi p \Gamma\left(\nu^{\prime}+i p+1\right) \Gamma(1-i p)}, \\
& \beta_{p}=\frac{e^{\pi p / 2}\left(e^{-\pi p}-\sigma e^{-i \pi \nu^{\prime}}\right)}{2 \sinh \pi p \Gamma\left(\nu^{\prime}-i p+1\right) \Gamma(1+i p)} .
\end{aligned}
$$

Then

$$
\begin{aligned}
N_{p \sigma}^{(2)} \delta\left(p-p^{\prime}\right)=\lim _{\epsilon \rightarrow 0}( & -\int_{\epsilon}^{0} \frac{d x}{x}\left\{\alpha_{p} \overline{\alpha_{p^{\prime}}} x^{i\left(p^{\prime}-p\right)}+\beta_{p} \overline{\beta_{p^{\prime}}} x^{i\left(p-p^{\prime}\right)}\right\} \\
& \left.+\int_{0}^{\epsilon} \frac{d x}{x}\left\{\alpha_{p} \overline{\alpha_{p^{\prime}}} x^{i\left(p^{\prime}-p\right)}+\beta_{p} \overline{\beta_{p^{\prime}}} x^{i\left(p-p^{\prime}\right)}\right\}\right) \\
= & 2 \pi\left\{\left|\alpha_{p}\right|^{2}+\left|\beta_{p}\right|^{2}\right\} \delta\left(p-p^{\prime}\right),
\end{aligned}
$$

from which we obtain

$$
N_{p \sigma}^{(2)}=\frac{2\left(\cosh \pi p-\sigma \cos \nu^{\prime} \pi\right)}{p \sinh \pi p\left|\Gamma\left(\nu^{\prime}+i p+1\right)\right|^{2}}
$$

From Eqs. (A5) and (A14), we finally find

$$
N_{p \sigma l m, p^{\prime} \sigma^{\prime} l^{\prime} m^{\prime}}=\frac{4\left(\cosh \pi p-\sigma \cos \nu^{\prime} \pi\right)}{\pi\left|\Gamma\left(\nu^{\prime}+i p+1\right)\right|^{2}} \delta\left(p-p^{\prime}\right) \delta_{\sigma \sigma^{\prime}} \delta_{l l^{\prime}} \delta_{m m^{\prime}}
$$

We see that this exactly coincides with the result obtained in Eq. (2.22).

\section{APPENDIX B:}

In this Appendix we show the outline of a proof of Eq. (4.7). After summing over $m$ in the left hand side of the equation, the relation to be proved becomes

$$
\begin{aligned}
\sum_{l=0}^{\infty} M_{l} & =\frac{P_{-\nu^{\prime}-1 / 2}^{-1 / 2}(\cosh \zeta)}{\sqrt{\sinh \zeta}} \lim _{r \rightarrow 0} \frac{P_{-\nu^{\prime}-1 / 2}^{-1 / 2}(\cosh r)}{\sqrt{\sinh r}}=\sqrt{\frac{2}{\pi}} \frac{P_{-\nu^{\prime}-1 / 2}^{-1 / 2}(\cosh \zeta)}{\sqrt{\sinh \zeta}}, \\
M_{l} & :=\frac{\Gamma\left(-\nu^{\prime}+l+1\right) \Gamma\left(\nu^{\prime}+l+1\right)}{\Gamma\left(-\nu^{\prime}+1\right) \Gamma\left(\nu^{\prime}+1\right)} \frac{P_{-\nu^{\prime}-1 / 2}^{-l-1 / 2}\left(\cosh r^{\prime}\right)}{\sqrt{\sinh r^{\prime}}} \frac{P_{-\nu^{\prime}-1 / 2}^{-l-1 / 2}\left(\cosh r^{\prime \prime}\right)}{\sqrt{\sinh r^{\prime \prime}}}(2 l+1) P_{l}(\cos \Theta),
\end{aligned}
$$


where

$$
\begin{aligned}
\cos \Theta & =\cos \theta^{\prime} \cos \theta^{\prime \prime}+\sin \theta^{\prime} \sin \theta^{\prime \prime} \cos \left(\phi^{\prime}-\phi^{\prime \prime}\right), \\
\cosh \zeta & =\cosh r^{\prime} \cosh r^{\prime \prime}-\sinh r^{\prime} \sinh r^{\prime \prime} \cos \Theta,
\end{aligned}
$$

and $P_{l}(\cos \Theta)$ is the Legendre function. Here we assume $\nu^{\prime}>0$ and $\nu^{\prime} \neq$ integer.

The strategy is similar to the technique employed in Section III. First we show that $\sum_{l} M_{l}$ converges. Then we show the result depends only on $\zeta$. Then taking the limit $r^{\prime \prime} \rightarrow 0$, it is readily seen that Eq. (B1) holds.

First let us show the convergence of $\sum_{l} M_{l}$. For this purpose, we rewrite $M_{l}$ as

$$
M_{l}=(2 l+1) \frac{\Gamma\left(-\nu^{\prime}+1\right) \Gamma\left(\nu^{\prime}+l+1\right)}{\Gamma\left(\nu^{\prime}+1\right) \Gamma\left(-\nu^{\prime}+l+1\right)} F_{l}\left(r^{\prime}\right) F_{l}\left(r^{\prime \prime}\right) P_{l}(\cos \Theta),
$$

where the function $F_{l}(r)$ is defined by

$$
\begin{aligned}
F_{l}(r) & :=\frac{\Gamma\left(-\nu^{\prime}+l+1\right)}{\Gamma\left(-\nu^{\prime}+1\right)} \frac{P_{-\nu^{\prime}-1 / 2}^{-l-1 / 2}(\cosh r)}{\sqrt{\sinh r}} \\
& =\sqrt{\frac{2}{\pi}} \Gamma\left(-\nu^{\prime}+1\right) \sinh y e^{i \pi \nu^{\prime}} Q_{l}^{-\nu^{\prime}}(\cosh y),
\end{aligned}
$$

and $y$ is related to $r$ as $\sinh y=1 / \sinh r$ or $y=\ln \operatorname{coth} r / 2$. With the aid of the integral representation,

$$
\begin{aligned}
e^{i \pi \nu^{\prime}} Q_{l}^{-\nu^{\prime}}(\cosh y)= & \frac{\sqrt{\pi} \sinh ^{-\nu^{\prime}} y}{\sqrt{2} \Gamma\left(\nu^{\prime}+1 / 2\right)} \int_{y}^{\infty} \frac{e^{-(l+1 / 2) x} d x}{(\cosh x-\cosh y)^{-\nu^{\prime}+1 / 2},} \\
& \text { for } \Re \nu^{\prime}>-1 / 2, \quad \Re\left(\nu^{\prime}-l\right)<1, \quad y>0,
\end{aligned}
$$

we see that $F_{l}(r)$ is real and positive for $l>\nu^{\prime}-1$. Further it forms a monotonically decreasing sequence for $l>\nu^{\prime}-1$. Hence it is bounded as

$$
0<F_{l}(r)<e^{-(l-n) y} F_{n}(r)=\left(\tanh \frac{r}{2}\right)^{l-n} F_{n}(r),
$$

for $l>n=\left[\nu^{\prime}\right]$ where $\left[\nu^{\prime}\right]$ is the largest integer not exceeding $\nu^{\prime}$. Also for the same $n$,

$$
\frac{\Gamma\left(\nu^{\prime}+l+1\right)}{\Gamma\left(-\nu^{\prime}+l+1\right)}<\left(\nu^{\prime}+l\right)\left(\nu^{\prime}+l-1\right) \cdots\left(\nu^{\prime}+l-2 n-1\right)<\left(\nu^{\prime}+l\right)^{2 n+2} .
$$

Thus, for sufficiently large $l$, the absolute value of $M_{l}$ is bounded from above as

$$
\left|M_{l}\right|<\frac{(2 l+1) \Gamma\left(-\nu^{\prime}+1\right)}{\Gamma\left(\nu^{\prime}+1\right)}\left(\nu^{\prime}+l\right)^{2 n+2}\left(\tanh \frac{r^{\prime}}{2} \tanh \frac{r^{\prime \prime}}{2}\right)^{l-n} F_{n}\left(r^{\prime}\right) F_{n}\left(r^{\prime \prime}\right),
$$

which becomes exponentially small for large $l$. Therefore the series $\sum_{l} M_{l}$ is manifestly convergent. In particular, if $0<\nu^{\prime}<1, F_{n}(r)$ is bounded from above for real positive $r$ and approaches zero as $r \rightarrow \infty$. Thus the series converges uniformly and absolutely for $0<\nu^{\prime}<1$. Furthermore, even for $\nu^{\prime}>1$, as long as we keep $r$ finite, say $r<r_{\text {max }}$, the series also converges uniformly and absolutely there. In this sense, the convergence is uniform for any $\nu^{\prime}>0$, $\nu^{\prime} \neq$ integer (uniform convergence on compact sets).

Next we show the series $\sum_{l} M_{l}$ is indeed a function of $\zeta$ alone. The equation to be proved is

$$
\sum_{l}\left(\cos ^{2} \Theta-1\right)\left\{\frac{\partial \zeta}{\partial \cosh r^{\prime}} \frac{\partial M_{l}}{\partial \cos \Theta}-\frac{\partial \zeta}{\partial \cos \Theta} \frac{\partial M_{l}}{\partial \cosh r^{\prime}}\right\}=0
$$

This calculation is a bit more complicated than the previous case in Section III because the counterpart of $e^{( \pm \nu-n) \zeta}$ there is $P_{l}(\cos \Theta)$ here. Taking derivatives with respect to $\cos \Theta$ gives terms as $\cos \Theta P_{l}(\cos \Theta)$ and $\cos ^{2} \Theta P_{l}(\cos \Theta)$. The factor $\cos \Theta$ must be removed from these terms by using the relation,

$$
\cos \Theta P_{l}(\cos \Theta)=\frac{1}{2 l+1}\left\{(l+1) P_{l+1}(\cos \Theta)+l P_{l-1}(\cos \Theta)\right\} .
$$


After carrying out this procedure, the left hand side of Eq. (B9) takes the form, $\sum_{l} C_{l}\left(r^{\prime}, r^{\prime \prime}\right) P_{l}(\cos \Theta)$. Then applying the recursion relation for the Associated Legendre functions, it can be shown that $C_{l}\left(r^{\prime}, r^{\prime \prime}\right)=0$. Thus the right hand side of Eq. (B1) has the desired invariance, which then immediately implies it is equivalent to the left hand side.

[1] See, e.g., S.D.M. White, in Clusters and Superclusters of Galaxies, ed. A.C. Fabian (Kluwer Academic Press, Netherlands, 1992), 17, and references cited therein.

[2] G. Smoot et al., Astrophys. J. 396, L1 (1992).

[3] D.H. Lyth and E.D. Stewart, Phys. Lett. B252, 336 (1990); B. Ratra and P.J.E. Peebles, Astrophys. J. Lett. 432, L5 (1994).

[4] See, e.g., D.N. Spergel, U-L. Pen, M. Kamionski and N. Sugiyama, in Relativistic Cosmology, Proceedings of the 8th Nishinomiya-Yukawa Memorial Symposium, ed. M. Sasaki (Universal Academy Press, Tokyo, 1994), 57, and references cited therein.

[5] N. Sugiyama and N. Gouda, Prog. Theor. Phys. 88, 803 (1992); W. Hu and N. Sugiyama, Phys. Rev. D50, 627 (1994).

[6] A. Kashlinsky, I.I. Tkachev and J. Friedman, Phys. Rev. Lett. 73, 1582 (1994).

[7] M. Sasaki, T. Tanaka, K. Yamamoto and J. Yokoyama, Phys. Lett. B317, 510 (1993)

[8] M. Bucher, A.S. Goldhaber and N. Turok, preprint iassns-hep-94-81, PUPT-94-1507, hep-ph/94411206.

[9] B. Allen and R. Caldwell, preprint WISC-MILW-94-TH-21.

[10] M. Sasaki, T. Tanaka and K. Yamamoto, preprint KUNS 1309.

[11] J.R. Gott, III, Nature 295, 304 (1982).

[12] S. Coleman, Phys. Rev. D15, 2929 (1977); C. Callan and S. Coleman, Phys. Rev. D16, 1763 (1977); S. Coleman and F. De Luccia, Phys. Rev. D21, 3305 (1980)

[13] V.A. Rubakov, Nucl. Phys. B245, 481 (1984).

[14] T. Vachaspati and A. Vilenkin, Phys. Rev. D43, 3846 (1991).

[15] T. Tanaka, M. Sasaki and K. Yamamoto, Phys. Rev. D 49, 1039 (1994).

[16] M. Sasaki, T. Tanaka, K. Yamamoto and J. Yokoyama, Prog. of Theor. Phys. 90, 1019 (1993).

[17] T. Tanaka and M. Sasaki, Phys. Rev. D, in press.

[18] K. Yamamoto, T. Tanaka and M. Sasaki, preprint KUNS 1305, gr-qc/9412011.

[19] T. Hamazaki, M. Sasaki, T. Tanaka and K. Yamamoto, preprint in preparation.

[20] E.A. Tagirov, Ann. Phys. 76, 561 (1973); T.S. Bunch and P.C. Davies, Proc. R. Soc. London A360, 117 (1978); B. Allen, Phys. Rev. D37, 2078 (1988).

[21] J.D. Pfautsch, Phys. Lett. 117B, 283 (1982).

[22] D. Gromes, H. J. Rothe and B. Stech, Nucl. Phys. B75, 313 (1973).

[23] The Legendre function $P$ and $Q$ correspond to $\mathcal{B}$ and $\mathcal{D}$, respectively, in a book by W. Magnus, F. Oberhettinger and R. P. Soni, Formulas and Theorems for the Special Functions of Mathematical Physics, (Springer-Verlag, Berlin Heidelberg, 1966).

\section{FIGURE CAPTIONS}

Fig. 1. Conformal diagram of de Sitter space with the coordinates $(t, r)=\left(t_{R}, r_{R}\right),\left(t_{L}, r_{L}\right)$ and $\left(t_{C}, r_{C}\right)$ spanning the regions $R, L$ and $C$, respectively. The thin real lines denote the surfaces $\{r=$ constant $\}$ and the broken lines $\{t=$ constant $\}$.

Fig. 2. Two different Cauchy surfaces on which the Klein-Gordon innner products are evaluated. One of them consists of hyperbolic time slices (I) and (II) in the regions $R$ and $L$, respectively, and the hypersurface (III) which connects them. The other one (IV) is an $r_{C}=$ constant hypersurface entirely contained in the region $C$.

Fig. 3. The contour of integration on the complex $u$-plane for Eq. (2.28). 
This figure "fig1-1.png" is available in "png" format from: http://arXiv.org/ps/gr-qc/9412025v1 


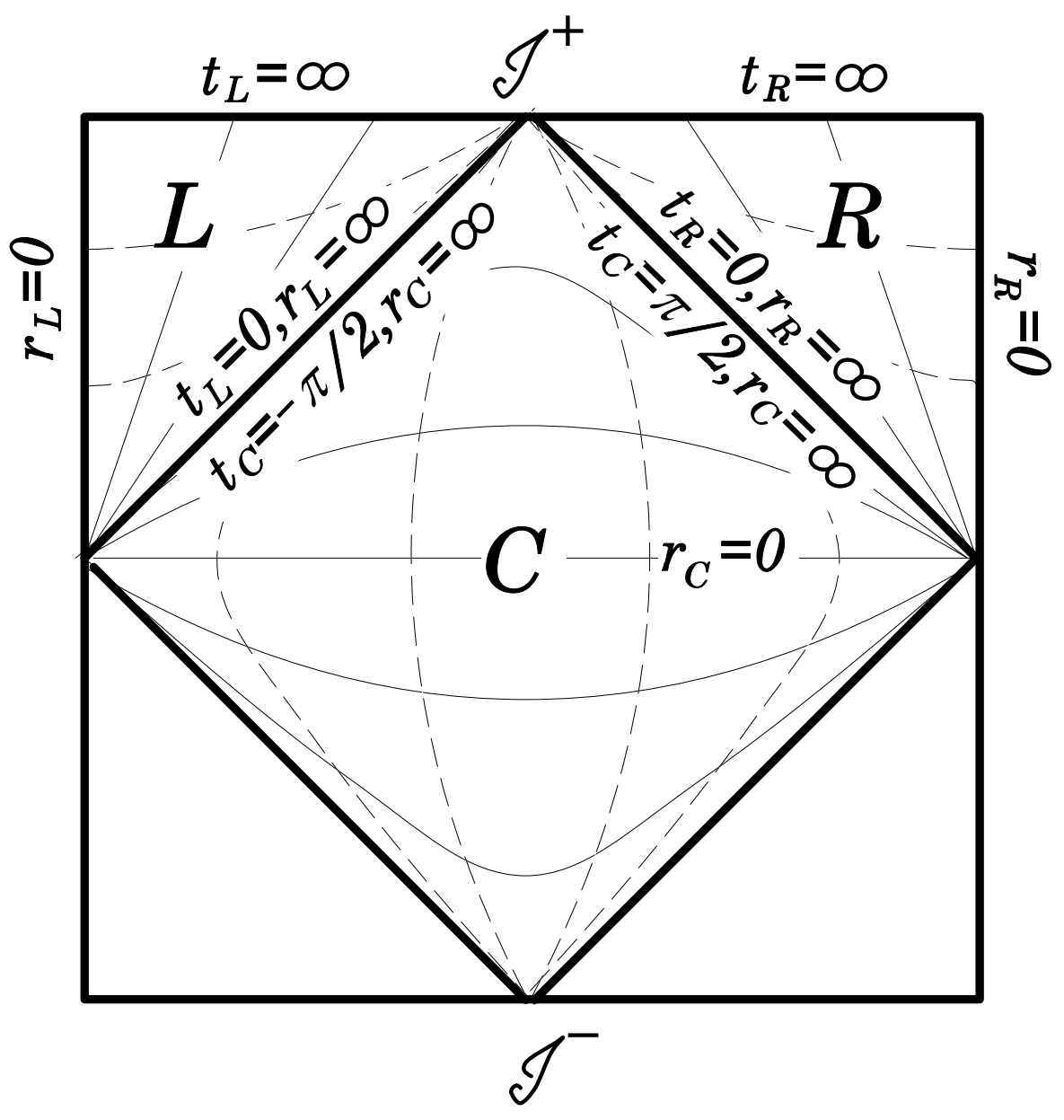

Fig. 1 
This figure "fig2-1.png" is available in "png" format from: http://arXiv.org/ps/gr-qc/9412025v1 
This figure "fig3-1.png" is available in "png" format from: http://arXiv.org/ps/gr-qc/9412025v1 


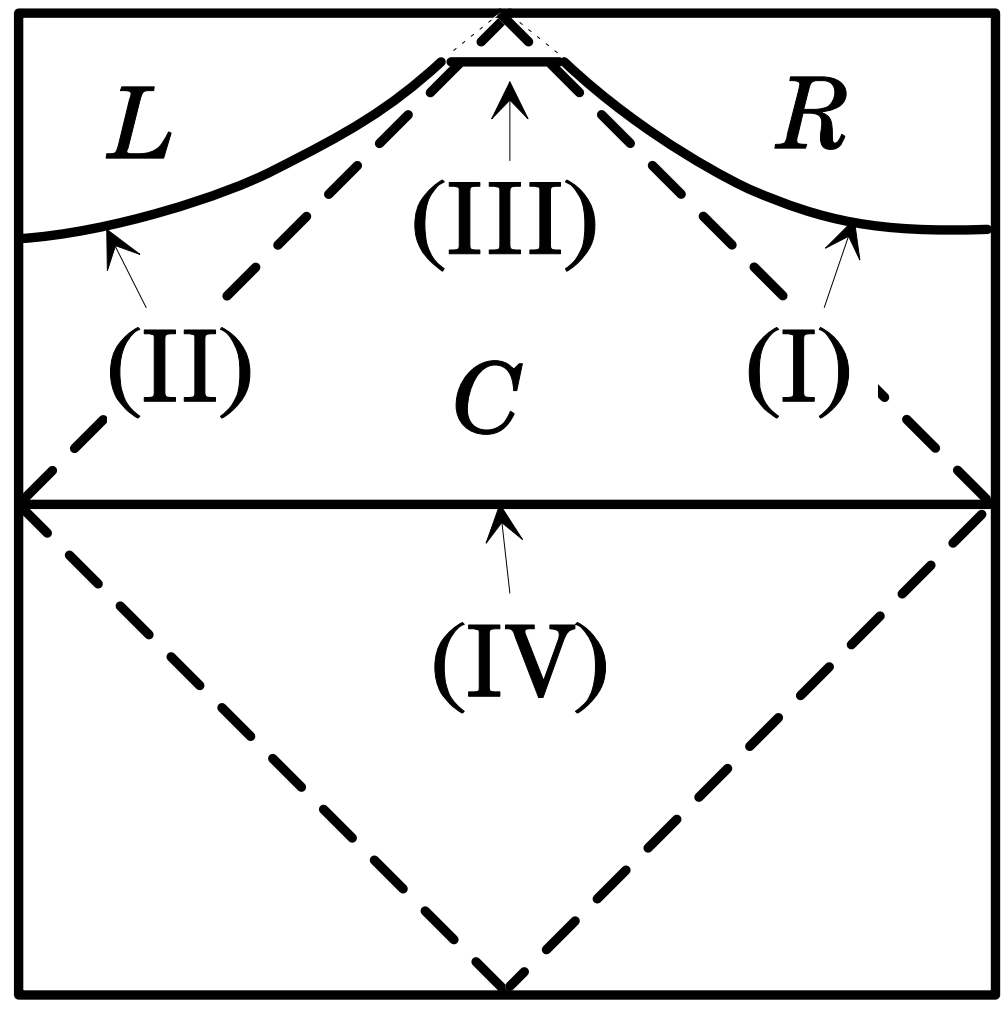

Fig. 2 


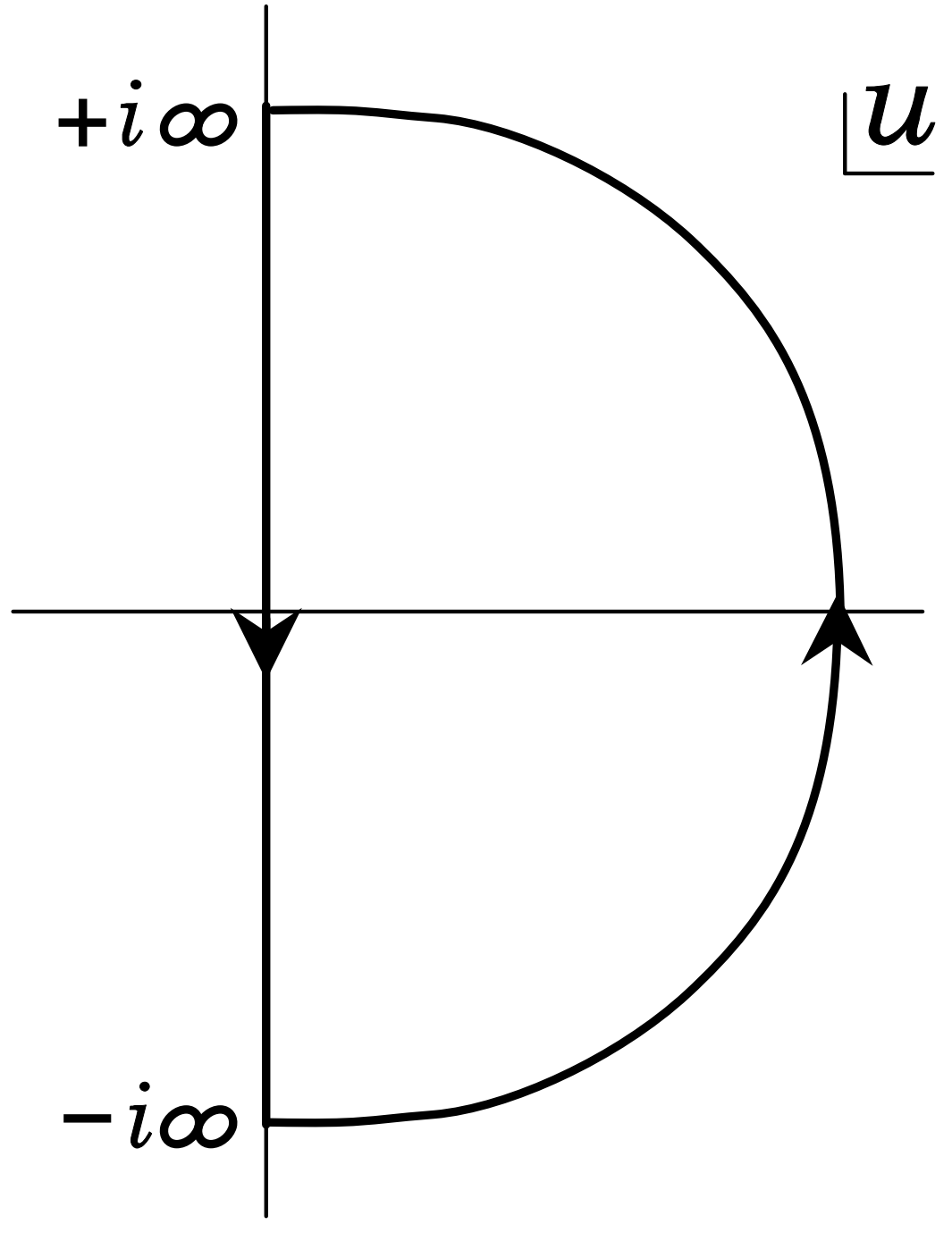

Fig. 3 